\title{
SUPERCONFORMAL ALGEBRAS AND MOCK THETA FUNCTIONS
}

\author{
TOHRU EGUCHI AND KAZUHIRO HIKAMI
}

\begin{abstract}
It is known that characters of BPS representations of extended superconformal algebras do not have good modular properties due to extra singular vectors coming from the BPS condition. In order to improve their modular properties we apply the method of Zwegers which has recently been developed to analyze modular properties of mock theta functions. We consider the case of $\mathcal{N}=4$ superconformal algebra at general levels and obtain the decomposition of characters of BPS representations into a sum of simple Jacobi forms and an infinite series of non-BPS representations.

We apply our method to study elliptic genera of hyper-Kähler manifolds in higher dimensions. In particular we determine the elliptic genera in the case of complex 4 dimensions of the Hilbert scheme of points on K3 surfaces $K^{[2]}$ and complex tori $A^{[[3]]}$.
\end{abstract}

\section{INTRODUCTION}

Superconformal field theory (SCFT) in two-dimensions provides a fundamental tool in describing string theory compactified on some target manifold $X$. One considers SCFT with an extended world-sheet $\mathcal{N}=2$ or 4 supersymmetry depending on whether the manifold $X$ is Kähler or hyper-Kähler. It is well-known that the special feature of SCFT's with extended SUSY is the existence of short or BPS representations in addition to the long or non-BPS representations. BPS representations appear at special values of conformal dimensions $h$ and describe massless states in compactified string theory which encode the geometrical information of target manifolds. On the other hand, non-BPS representations appear at continuous values of $h$ and correspond to massive excitations in string theory. As we shall see, characters of BPS representations in general do not have a simple transformation law under modular transformations: this is due to the denominator factors which come from the BPS condition that the supercharges annihilate BPS states.

In this paper we would like to propose a method which replace characters of BPS representations by some Jacobi forms, i.e. forms with good modular properties obtained by adding an infinite series of characters of non-BPS representations. This construction is based on an analogy with Zwegers' treatment of mock theta functions where mock theta functions are replaced by (real analytic) Jacobi forms by the addition of Eichler integral of suitable "shadows" of mock theta functions.

Date: December 5, 2008. Revised on February 27, 2009.

2000 Mathematics Subject Classification.

Key words and phrases. 
In the following we restrict our discussions to the case of $\mathcal{N}=4$ SCFT, however, very similar structures emerge also in $\mathcal{N}=2$ case.

Study of the $\mathcal{N}=4$ superconformal field theory has been initiated some time ago in [8, 9, 10]. $\mathcal{N}=4$ superconformal algebra (SCA) contains, besides the energy-momentum tensor, 4 supercurrents and a triplet of currents which constitute the affine Lie algebra $S U(2)_{k}$. There exits two types of representations, i.e. BPS and non-BPS representation. We call their character formulas as massless and massive characters, respectively. Complexity of modular properties of massless characters has been noticed in [5, 6] and the level- $k=1$ case has been considered in detail in connection with the geometry of $K 3$ surface.

The difficulty of the massless character comes from the fact that it has the form of the Lerch sum as in (2.3). Lerch sum has been long studied in analytic number theory in connection with the so-called mock theta functions which first appeared in Ramanujan's last letter to Hardy in 1920 (see e.g., Ref. [1]). Although mock theta function is not modular, it has a quasi modular property as was first shown by Watson [24]. Underlying intrinsic mathematical structure of these functions has recently been clarified by Zwegers in his thesis [27]; the mock theta function is a holomorphic part of the harmonic Maass form with weight $1 / 2$, which is a Jacobi form and an eigenfunction of the second order differential operator. As an application, Bringmann and Ono resolved a rank-generating problem of integer partitions [4] by constructing the Poincaré-Maass series [3].

The purpose of this paper is to apply Zwegers' method to the $\mathcal{N}=4$ superconformal algebras at general level- $k$ and construct a Jacobi form for each massless representation. We show that the construction of Jacobi form is useful in decomposing elliptic genus in terms of the irreducible superconformal representations. As an application we will determine the elliptic genera of hyper-Kähler manifolds in complex 4-dimensions, in particular the Hilbert scheme of points on $K 3$ surfaces $K 3^{[2]}$ and complex tori $A^{[3]]}$.

This paper is organized as follows. In Section 2, we briefly review the representation theory and character formulas of $\mathcal{N}=4 \mathrm{SCA}$. We reconsider the level-1 case using Zwegers' formalism in Section [3. In Section 4 we study in detail the higher-level $\mathcal{N}=4$ SCFT and present elliptic genera of complex 4-dimensional hyper-Kähler manifolds. Definitions of theta functions and numerical data of some expansion coefficients are relegated to Appendices $\mathrm{A}$ and $\mathrm{B}$.

\section{Superconformal Algebra and Elliptic Genus}

2.1. Character of Superconformal Algebra. The $\mathcal{N}=4$ superconformal algebra with central charge $c=6 k$ contains a level- $k$ affine $\mathrm{SU}(2)$ algebra. The highest weight states are labeled by the conformal dimension $h$ and isospin $\ell$, satisfying $0 \leq \ell \leq k / 2$ with $\ell \in \mathbb{Z} / 2$. 
Due to the unitarity, we have a bound on conformal dimension in R and NS sector as

$$
\begin{cases}\mathrm{R}: & h \geq \frac{k}{4}, \\ \mathrm{NS}: & h \geq \ell .\end{cases}
$$

In each of these sectors, there exist two types of representations [8, 9, 10];

- massive (non-BPS) representation,

$$
\left\{\begin{array}{lll}
\mathrm{R}: & h>\frac{k}{4}, & \ell=\frac{1}{2}, 1, \ldots, \frac{k}{2} \\
\mathrm{NS}: & h>\ell, & \ell=0, \frac{1}{2}, \ldots, \frac{k-1}{2},
\end{array}\right.
$$

- massless (BPS) representation,

$$
\left\{\begin{array}{lll}
\mathrm{R}: & h=\frac{k}{4}, & \ell=0, \frac{1}{2}, \ldots, \frac{k}{2}, \\
\mathrm{NS}: & h=\ell, & \ell=0, \frac{1}{2}, \ldots, \frac{k}{2} .
\end{array}\right.
$$

The character is a trace over the representation space

$$
\operatorname{ch}_{k, h, \ell}(z ; \tau)=\operatorname{Tr}_{\mathcal{H}}\left(\mathrm{e}^{2 \pi \mathrm{i} z T_{0}^{3}} q^{L_{0}-\frac{c}{24}}\right),
$$

where $q=\mathrm{e}^{2 \pi \mathrm{i} \tau}$ with $\tau \in \mathbb{H}$ as usual. $L_{0}$ and $T_{0}^{3}$ denote the zero mode of Virasoro operator and the 3rd component of the $S U(2)$ current, respectively. The characters in the Ramond sector, for instance, are computed as [ $[\mathbf{8},[9]$

- massive character,

$$
\operatorname{ch}_{k, h, \ell}^{R}(z ; \tau)=q^{h-\frac{\ell^{2}}{k+1}-\frac{k}{4}} \frac{\left[\theta_{10}(z ; \tau)\right]^{2}}{[\eta(\tau)]^{3}} \chi_{k-1, \ell-\frac{1}{2}}(z ; \tau),
$$

- massless character,

$$
\begin{aligned}
\operatorname{ch}_{k, \frac{k}{4}, \ell}^{R}(z ; \tau)=\frac{\mathrm{i}}{\theta_{11}(2 z ; \tau)} \cdot \frac{\left[\theta_{10}(z ; \tau)\right]^{2}}{[\eta(\tau)]^{3}} & \\
& \times \sum_{\varepsilon= \pm 1} \sum_{m \in \mathbb{Z}} \varepsilon \frac{\mathrm{e}^{4 \pi \mathrm{i} \varepsilon((k+1) m+\ell) z}}{\left(1+\mathrm{e}^{-2 \pi \mathrm{i} \varepsilon z} q^{-m}\right)^{2}} q^{(k+1) m^{2}+2 \ell m} .
\end{aligned}
$$

See Appendix $\AA$ for definitions of theta functions. $\chi_{k, \ell}(z ; \tau)$ denotes the character of the spin $\ell$ representation of $S U(2)_{k}$ algebra. Note that the denominator in the massless character originates from the BPS condition that the supercharge annihilates the BPS states. If we ignore the denominator $\left(1+\mathrm{e}^{-2 \pi \mathrm{i} \varepsilon z} q^{-m}\right)^{2}$ in the massless character, it takes the same form as the massive character at $h=k / 4$. We can check directly that the massless characters (2.3) satisfy a recursion relation

$$
\begin{aligned}
\operatorname{ch}_{k, \frac{k}{4}, \ell}^{R}(z ; \tau)+2 \operatorname{ch}_{k, \frac{k}{4}, \ell-\frac{1}{2}}^{R}(z ; \tau)+\operatorname{ch}_{k, \frac{k}{4}, \ell-1}^{R}(z ; \tau) & \\
& =q^{-\frac{\ell^{2}}{k+1}} \frac{\left[\theta_{10}(z ; \tau)\right]^{2}}{[\eta(\tau)]^{3}} \chi_{k-1, \ell-\frac{1}{2}}(z ; \tau) .
\end{aligned}
$$


Note that the right hand side corresponds to the character of massive representation in the limit,

$$
\lim _{h \searrow \frac{k}{4}} \operatorname{ch}_{k, h, \ell}^{R}(z ; \tau)=q^{-\frac{\ell^{2}}{k+1}} \frac{\left[\theta_{10}(z ; \tau)\right]^{2}}{[\eta(\tau)]^{3}} \chi_{k-1, \ell-\frac{1}{2}}(z ; \tau),
$$

and (2.4) shows how the non-BPS representation decomposes into a sum of BPS representations at the unitarity bound.

The characters in the NS sector are given from those in the R sector by the spectral flow as

$$
\operatorname{ch}_{k, h+\frac{\ell}{2}+\frac{k}{4}, \frac{k}{2}-\ell}^{N S}(z ; \tau)=q^{\frac{k}{4}} \mathrm{e}^{2 \pi \mathrm{i} k z} \operatorname{ch}_{k, h, \ell}^{R}\left(z+\frac{\tau}{2} ; \tau\right) .
$$

Characters of other sectors are also defined by

$$
\begin{aligned}
& \operatorname{ch}_{k, h, \ell}^{\widetilde{R}}(z ; \tau)=\operatorname{ch}_{k, h, \ell}^{R}\left(z+\frac{1}{2} ; \tau\right), \\
& \operatorname{ch}_{k, h, \ell}^{\widetilde{N S}}(z ; \tau)=\operatorname{ch}_{k, h, \ell}^{N S}\left(z+\frac{1}{2} ; \tau\right) .
\end{aligned}
$$

2.2. Elliptic Genus. When the superconformal field theory is interpreted as a nonlinear sigma model on the Calabi-Yau manifold $X$ with a complex dimension $c / 3$, the elliptic genus of $X$ is identified with [25]

$$
Z_{X}(z ; \tau)=\operatorname{Tr}_{\mathcal{H}^{R} \otimes \mathcal{H}^{R}}(-1)^{F} \mathrm{e}^{2 \pi \mathrm{i} T_{0}^{3} z} q^{L_{0}-\frac{c}{24}} \bar{q}^{\bar{L}_{0}-\frac{c}{24}},
$$

where $(-1)^{F}=\mathrm{e}^{\pi \mathrm{i}\left(T_{0}^{3}-\bar{T}_{0}^{3}\right)}$, and $\mathcal{H}^{R}$ denotes Hilbert space of the Ramond sector. Due to the supersymmetry, the elliptic genus is independent of $\bar{q}$, and only the ground states contributes in the right-moving sector. The fundamental structure of the elliptic genus $Z_{X}(z ; \tau)$ is the modular property [11, 16];

$$
\begin{gathered}
Z_{X}\left(\frac{z}{\tau} ;-\frac{1}{\tau}\right)=\mathrm{e}^{2 \pi \mathrm{i} \frac{c}{6} \frac{z^{2}}{\tau}} Z_{X}(z ; \tau), \\
Z_{X}(z ; \tau+1)=Z_{X}(z ; \tau), \\
Z_{X}(z+1 ; \tau)=(-1)^{\frac{c}{3}} Z_{X}(z ; \tau), \\
Z_{X}(z+\tau ; \tau)=(-1)^{\frac{c}{3}} q^{-\frac{c}{6}} \mathrm{e}^{-2 \pi \mathrm{i} \frac{c}{3} z} Z_{X}(z ; \tau) .
\end{gathered}
$$

It is known that the elliptic genus at special values of $z$ gives classical topological invariants of $X[5]$;

$$
\begin{gathered}
Z_{X}(z=0 ; \tau)=\chi_{X}, \\
Z_{X}\left(z=\frac{1}{2} ; \tau\right)=\sigma_{X}+\mathcal{O}(q), \\
q^{\frac{c}{12}} Z_{X}\left(z=\frac{1+\tau}{2} ; \tau\right)=\widehat{A}_{X}+\mathcal{O}(q),
\end{gathered}
$$


where $\chi_{X}, \sigma_{X}$, and $\widehat{A}_{X}$ are respectively the Euler characteristic, the Hirzebruch signature, and the $\widehat{A}$-genus of $X$.

\section{Level $k=1$ Superconformal Algebra Revisited}

The massless character in the $\widetilde{R}$-sector is read from (2.3) as

$$
\operatorname{ch}_{k=1, h=\frac{1}{4}, \ell=0}^{\widetilde{R}}(z ; \tau)=\frac{\mathrm{i}}{\theta_{11}(2 z ; \tau)} \cdot \frac{\left[\theta_{11}(z ; \tau)\right]^{2}}{[\eta(\tau)]^{3}} \sum_{m \in \mathbb{Z}} q^{2 m^{2}} \mathrm{e}^{8 \pi \mathrm{i} m z} \frac{1+\mathrm{e}^{2 \pi \mathrm{i} z} q^{m}}{1-\mathrm{e}^{2 \pi \mathrm{i} z} q^{m}},
$$

and the identity (2.4) reduces to

$$
\operatorname{ch}_{k=1, h=\frac{1}{4}, \ell=\frac{1}{2}}^{\widetilde{R}}(z ; \tau)+2 \operatorname{ch}_{k=1, h=\frac{1}{4}, \ell=0}^{\widetilde{R}}(z ; \tau)=q^{-\frac{1}{8}} \frac{\left[\theta_{11}(z ; \tau)\right]^{2}}{[\eta(\tau)]^{3}} .
$$

The character (3.1) is known to be rewritten as [10]

$$
\operatorname{ch}_{k=1, h=\frac{1}{4}, \ell=0}^{\widetilde{R}}(z ; \tau)=\frac{\left[\theta_{11}(z ; \tau)\right]^{2}}{[\eta(\tau)]^{3}} \mu(z ; \tau),
$$

where

$$
\mu(z ; \tau)=\frac{\mathrm{i} \mathrm{e}^{\pi \mathrm{i} z}}{\theta_{11}(z ; \tau)} \sum_{n \in \mathbb{Z}}(-1)^{n} \frac{q^{\frac{1}{2} n(n+1)} \mathrm{e}^{2 \pi \mathrm{in} z}}{1-q^{n} \mathrm{e}^{2 \pi \mathrm{i} z}} .
$$

We reconsider the modular properties of $\mu(z ; \tau)$ using Zwegers' approach.

3.1. Harmonic Maass Form. Zwegers defines the function [27]

$$
\mu(u, v ; \tau)=\frac{\mathrm{i}^{\pi \mathrm{i} u}}{\theta_{11}(v ; \tau)} \sum_{n \in \mathbb{Z}} \frac{\left(-\mathrm{e}^{2 \pi \mathrm{i} v}\right)^{n} q^{\frac{1}{2} n(n+1)}}{1-\mathrm{e}^{2 \pi \mathrm{i} u} q^{n}},
$$

which is known as the Lerch sum (or, generalized Lambert series). $\mu(z ; \tau)$ of (3.4) and $\mu(u, v ; \tau)$ are simply related as $\mu(u=z, v=z ; \tau)=\mu(z ; \tau)$. Zwegers showed that, under the $S$-transformation, $\mu(u, v ; \tau)$ behaves as

$$
\mu(u, v ; \tau)+\sqrt{\frac{\mathrm{i}}{\tau}} \mathrm{e}^{\pi \mathrm{i} \frac{(u-v)^{2}}{\tau}} \mu\left(\frac{u}{\tau}, \frac{v}{\tau} ;-\frac{1}{\tau}\right)=\frac{1}{2} M(u-v ; \tau),
$$

where $M(x ; \tau)$ is the Mordell integral [18] defined by

$$
M(x ; \tau)=\int_{-\infty}^{\infty} \frac{\mathrm{e}^{\pi \mathrm{i} \tau z^{2}-2 \pi x z}}{\cosh (\pi z)} \mathrm{d} z .
$$

He further introduced a non-holomorphic partner of $\mu(u, v ; \tau)$ by

$$
\begin{aligned}
R(z ; \tau)=\sum_{n \in \mathbb{Z}}(-1)^{n}\left[\operatorname{sgn}\left(n+\frac{1}{2}\right)-E\left(\left(n+\frac{1}{2}+\frac{\Im z}{\Im \tau}\right)\right.\right. & \sqrt{2 \Im \tau})] \\
& \times \mathrm{e}^{-2 \pi \mathrm{i}\left(n+\frac{1}{2}\right) z} q^{-\frac{1}{2}\left(n+\frac{1}{2}\right)^{2}},
\end{aligned}
$$


where $E(z)$ is the error function defined by

$$
E(z)=2 \int_{0}^{z} \mathrm{e}^{-\pi u^{2}} \mathrm{~d} u=1-\operatorname{erfc}(\sqrt{\pi} z)
$$

The non-holomorphic function is shown to fulfill the $S$-transformation

$$
R(u ; \tau)+\sqrt{\frac{\mathrm{i}}{\tau}} \mathrm{e}^{\pi \mathrm{i} \frac{u^{2}}{\tau}} R\left(\frac{u}{\tau} ;-\frac{1}{\tau}\right)=M(u ; \tau) .
$$

Zwegers combined these two functions to define

$$
\widehat{\mu}(u, v ; \tau)=\mu(u, v ; \tau)-\frac{1}{2} R(u-v ; \tau),
$$

as a completion of the Lerch sum $\mu(u, v ; \tau)$. The Mordell integral $M(u ; \tau)$ disappears in the $S$-transformation of $\widehat{\mu}(u, v ; \tau)$, and it behaves like a 2 -variable Jacobi form with weight $1 / 2$ [11]

$$
\begin{aligned}
\widehat{\mu}(u, v ; \tau) & =-\sqrt{\frac{\mathrm{i}}{\tau}} \mathrm{e}^{\pi \mathrm{i} \frac{(u-v)^{2}}{\tau}} \widehat{\mu}\left(\frac{u}{\tau}, \frac{v}{\tau} ;-\frac{1}{\tau}\right) \\
& =\mathrm{e}^{\pi \mathrm{i} / 4} \widehat{\mu}(u, v ; \tau+1) .
\end{aligned}
$$

In view of (3.4) and (3.5), we see that the massless character (3.3) is proportional to the holomorphic part of the real analytic modular form

$$
\widehat{\mu}(z ; \tau)=\mu(z ; \tau)-\frac{1}{2} R(0 ; \tau)
$$

whose transformation formulae are

$$
\begin{gathered}
\widehat{\mu}(z ; \tau)=-\sqrt{\frac{\mathrm{i}}{\tau}} \widehat{\mu}\left(\frac{z}{\tau} ;-\frac{1}{\tau}\right), \\
\widehat{\mu}(z ; \tau+1)=\mathrm{e}^{-\frac{1}{4} \pi \mathrm{i}} \widehat{\mu}(z ; \tau), \\
\widehat{\mu}(z+1 ; \tau)=\widehat{\mu}(z+\tau ; \tau)=\widehat{\mu}(z ; \tau) .
\end{gathered}
$$

It is possible to show that the non-holomorphic part $R(0 ; \tau)$ in (3.13) is an incomplete period integral of the modular form $[\eta(\tau)]^{3}$ with weight $3 / 2$;

$$
\text { i } R(0 ; \tau)=\int_{-\bar{\tau}}^{\mathrm{i} \infty} \frac{[\eta(z)]^{3}}{\sqrt{\frac{z+\tau}{\mathrm{i}}}} \mathrm{d} z,
$$

which shows that the massless character (3.3) of the level-1 superconformal algebra has a modular form $[\eta(\tau)]^{3}$ as a "shadow" in the sense of Zagier [26]. We then see that the real analytic function $\widehat{\mu}(z ; \tau)$ satisfies

$$
\frac{\partial}{\partial \bar{\tau}} \widehat{\mu}(z ; \tau)=\frac{\mathrm{i}}{2} \frac{[\eta(-\bar{\tau})]^{3}}{\sqrt{2 \Im \tau}} .
$$

To conclude, we see that the function $\widehat{\mu}(z ; \tau)$ is a harmonic Maass form being a solution of the differential equation

$$
(\Im \tau)^{\frac{3}{2}} \frac{\partial}{\partial \tau} \sqrt{\Im \tau} \frac{\partial}{\partial \bar{\tau}} \widehat{\mu}(z ; \tau)=0
$$


which, with $\tau=u+\mathrm{i} v$, reduces to

$$
\left[-v^{2}\left(\frac{\partial^{2}}{\partial u^{2}}+\frac{\partial^{2}}{\partial v^{2}}\right)+\frac{\mathrm{i}}{2} v\left(\frac{\partial}{\partial u}+\mathrm{i} \frac{\partial}{\partial v}\right)\right] \widehat{\mu}(z ; \tau)=0 .
$$

We notice that the formula under the $S$-transformation (3.6) gives [10]

$$
\frac{\mu}{\eta}(z ; \tau)+\frac{\mu}{\eta}\left(\frac{z}{\tau} ;-\frac{1}{\tau}\right)=\frac{1}{\eta(\tau)} \int_{-\infty}^{\infty} \frac{\mathrm{e}^{\pi \mathrm{i} \tau x^{2}}}{2 \cosh (\pi x)} \mathrm{d} x
$$

3.2. Character Decomposition of Elliptic Genus. Let the function $J(z ; w ; \tau)$ be

$$
\begin{aligned}
J(z ; w ; \tau) & =\frac{\left[\theta_{11}(z ; \tau)\right]^{2}}{[\eta(\tau)]^{3}}(\widehat{\mu}(z ; \tau)-\widehat{\mu}(w ; \tau)) \\
& =\frac{\left[\theta_{11}(z ; \tau)\right]^{2}}{[\eta(\tau)]^{3}}(\mu(z ; \tau)-\mu(w ; \tau)) \\
& =\operatorname{ch}_{k=1, h=\frac{1}{4}, \ell=0}^{\widetilde{R}}(z ; \tau)-\frac{\left[\theta_{11}(z ; \tau)\right]^{2}}{[\eta(\tau)]^{3}} \mu(w ; \tau),
\end{aligned}
$$

where the second equality follows from the $z$-independence of $R(0 ; \tau)(\underline{3.13})$. We see that

$$
J(w ; w ; \tau)=0,
$$

and that $J(0 ; w ; \tau)=1$. By use of (3.14), we obtain the transformation formulae as follows;

$$
\begin{gathered}
J(z ; w ; \tau)=\mathrm{e}^{-2 \pi \mathrm{i} \frac{z^{2}}{\tau}} J\left(\frac{z}{\tau} ; \frac{w}{\tau} ;-\frac{1}{\tau}\right) \\
J(z+1 ; w ; \tau)=J(z ; w ; \tau+1)=J(z ; w+\tau ; \tau)=J(z ; w ; \tau), \\
J(z+\tau ; w ; \tau)=q^{-1} \mathrm{e}^{-4 \pi \mathrm{i} z} J(z ; w ; \tau) .
\end{gathered}
$$

The second equality of (3.20) indicates that the function $J(z ; w ; \tau)$ is a holomorphic function; when we fix $w$ to be specific values $w=\frac{1}{2}, \frac{1+\tau}{2}$, and $\frac{\tau}{2}$, those modular properties show

$$
J\left(z ; \frac{1}{2} ; \tau\right)=\left(\frac{\theta_{10}(z ; \tau)}{\theta_{10}(0 ; \tau)}\right)^{2}, J\left(z ; \frac{1+\tau}{2} ; \tau\right)=\left(\frac{\theta_{00}(z ; \tau)}{\theta_{00}(0 ; \tau)}\right)^{2}, J\left(z ; \frac{\tau}{2} ; \tau\right)=\left(\frac{\theta_{01}(z ; \tau)}{\theta_{01}(0 ; \tau)}\right)^{2}
$$

Lerch sums introduced in [10] are given by the values of $\mu(w ; \tau)$ at $w=1 / 2,1+\tau / 2, \tau / 2$

$$
\begin{gathered}
h_{2}(\tau) \equiv \frac{\mu}{\eta}\left(\frac{1}{2} ; \tau\right)=\frac{1}{\eta(\tau) \theta_{10}(0 ; \tau)} \sum_{n \in \mathbb{Z}} \frac{q^{\frac{1}{2} n(n+1)}}{1+q^{n}}, \\
h_{3}(\tau) \equiv \frac{\mu}{\eta}\left(\frac{1+\tau}{2} ; \tau\right)=\frac{1}{\eta(\tau) \theta_{00}(0 ; \tau)} \sum_{n \in \mathbb{Z}} \frac{q^{\frac{1}{2} n^{2}-\frac{1}{8}}}{1+q^{n-\frac{1}{2}}}, \\
h_{4}(\tau) \equiv \frac{\mu}{\eta}\left(\frac{\tau}{2} ; \tau\right)=\frac{1}{\eta(\tau) \theta_{01}(0 ; \tau)} \sum_{n \in \mathbb{Z}}(-1)^{n} \frac{q^{\frac{1}{2} n^{2}-\frac{1}{8}}}{1+q^{n-\frac{1}{2}}} .
\end{gathered}
$$


We then obtain the decomposition formula for the massless character

$$
\begin{aligned}
\operatorname{ch}_{k=1, h=\frac{1}{4}, \ell=0}^{\widetilde{R}}(z ; \tau) & =\left(\frac{\theta_{10}(z ; \tau)}{\theta_{10}(0 ; \tau)}\right)^{2}+h_{2}(\tau) \frac{\left[\theta_{11}(z ; \tau)\right]^{2}}{[\eta(\tau)]^{2}}, \\
& =\left(\frac{\theta_{00}(z ; \tau)}{\theta_{00}(0 ; \tau)}\right)^{2}+h_{3}(\tau) \frac{\left[\theta_{11}(z ; \tau)\right]^{2}}{[\eta(\tau)]^{2}}, \\
& =\left(\frac{\theta_{01}(z ; \tau)}{\theta_{01}(0 ; \tau)}\right)^{2}+h_{4}(\tau) \frac{\left[\theta_{11}(z ; \tau)\right]^{2}}{[\eta(\tau)]^{2}}
\end{aligned}
$$

Identities (3.25) are used to express the elliptic genus of K3 surface in terms of irreducible representations of $\mathcal{N}=4$ algebra. The elliptic genus of $\mathrm{K} 3$ surface is known to be given by [5, 16]

$$
Z_{K 3}(z ; \tau)=8\left[\left(\frac{\theta_{10}(z ; \tau)}{\theta_{10}(0 ; \tau)}\right)^{2}+\left(\frac{\theta_{00}(z ; \tau)}{\theta_{00}(0 ; \tau)}\right)^{2}+\left(\frac{\theta_{01}(z ; \tau)}{\theta_{01}(0 ; \tau)}\right)^{2}\right]
$$

Using (3.25), we can rewrite the elliptic genus as

$$
Z_{K 3}(z ; \tau)=24 \operatorname{ch}_{k=1, h=\frac{1}{4}, \ell=0}^{\widetilde{R}}(z ; \tau)-8\left[\frac{\theta_{11}(z ; \tau)}{\eta(\tau)}\right]^{2} \sum_{a=2,3,4} h_{a}(\tau) .
$$

We note that

$$
\begin{aligned}
8 \sum_{w \in\left\{\frac{1}{2}, \frac{1+\tau}{2}, \frac{\tau}{2}\right\}} \mu(w ; \tau) & =8 \eta(\tau) \sum_{a=2,3,4} h_{a}(\tau) \\
& =q^{-\frac{1}{8}}\left(2-\sum_{n \geq 1} A_{n} q^{n}\right),
\end{aligned}
$$

where $A_{n}$ are positive integers;

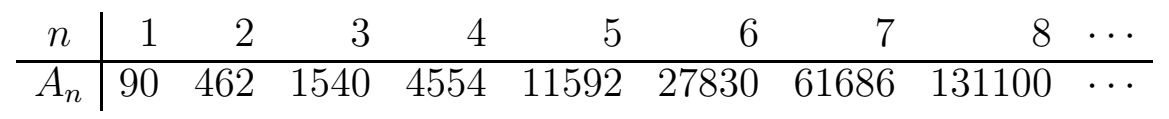

Using the relation (3.2), we obtain

$$
Z_{K 3}(z ; \tau)=20 \operatorname{ch}_{1, \frac{1}{4}, 0}^{\widetilde{R}}(z ; \tau)-2 \operatorname{ch}_{1, \frac{1}{4}, \frac{1}{2}}^{\widetilde{R}}(z ; \tau)+\sum_{n \geq 1} A_{n} \operatorname{ch}_{1, n+\frac{1}{4}, \frac{1}{2}}^{\widetilde{R}}(z ; \tau),
$$

where the first two terms are massless characters and the last term gives an infinite sum of massive characters. Note that the right-moving sector in the elliptic genus (2.8) gives a degeneracy factor $2 \ell+1$ for isospin $\ell$ representation. Hence the net multiplicity of isospin $1 / 2$ massless representation in (3.28) equals 1 . Since isospin $1 / 2$ representation in $\mathrm{R}$ sector flows to isospin 0 in NS sector, (3.28) has the multiplicity 1 for isospin 0 or vacuum representation in NS sector. 


\section{Higher Level Superconformal Algebra}

Massless character at level- $k$ with isospin-0 in the $\widetilde{R}$-sector is read from (2.3) as

$$
\operatorname{ch}_{k, \frac{k}{4}, \ell=0}^{\widetilde{R}}(z ; \tau)=\frac{\mathrm{i}}{\theta_{11}(2 z ; \tau)} \cdot \frac{\left[\theta_{11}(z ; \tau)\right]^{2}}{[\eta(\tau)]^{3}} \sum_{m \in \mathbb{Z}} \mathrm{e}^{4 \pi \mathrm{i}(k+1) m z} q^{(k+1) m^{2}} \frac{1+\mathrm{e}^{2 \pi \mathrm{i} z} q^{m}}{1-\mathrm{e}^{2 \pi \mathrm{i} z} q^{m}},
$$

and the recursion relation (2.4) of massless characters becomes

$$
\begin{aligned}
\operatorname{ch}_{k, \frac{k}{4}, \ell}^{\widetilde{R}}(z ; \tau)+2 \operatorname{ch}_{k, \frac{k}{4}, \ell-\frac{1}{2}}^{\widetilde{R}}(z ; \tau)+\operatorname{ch}_{k, \frac{k}{4}, \ell-1}^{\widetilde{R}}(z ; \tau) & \\
& =(-1)^{2 \ell+1} q^{-\frac{\ell^{2}}{k+1}} \frac{\left[\theta_{11}(z ; \tau)\right]^{2}}{[\eta(\tau)]^{3}} \chi_{k-1, \ell-\frac{1}{2}}(z ; \tau) .
\end{aligned}
$$

The modular transformation properties of (4.1) are studied [7] by use of the formula of Miki [17] and also the higher-level Appell function [20, 23]. We shall show that these massless characters are the mock theta functions whose "shadow" [26] is $\Psi_{k+1}^{(a)}(\tau)$ (A.10) related to the affine $\mathrm{SU}(2)$ characters at level- $k$. It is also related to the colored Jones polynomial for torus link $T(2,2 P)$ [12], and is a building block for the $\mathrm{SU}(2)$ Witten-Reshetikhin-Turaev invariant for the Seifert manifolds associated with the ADE singularities [13, 14].

4.1. Setup of Zwegers. We first review the results of Zwegers [27]. We introduce the Lerch sum as

$$
f_{P}(u, z ; \tau)=\sum_{n \in \mathbb{Z}} \frac{q^{P n^{2}} \mathrm{e}^{4 \pi \mathrm{i} P n z}}{1-q^{n} \mathrm{e}^{2 \pi \mathrm{i}(z-u)}},
$$

where $P \in \mathbb{Z}_{>0}$, and it is related to the level- $k$ of the superconformal algebra as $k=P-1$. Zwegers proved the following formulae;

$$
\begin{gathered}
f_{P}(u+1, z ; \tau)=f_{P}(u, z ; \tau), \\
f_{P}(u, z ; \tau)-q^{-P} \mathrm{e}^{-4 \pi \mathrm{i} P u} f_{P}(u+\tau, z ; \tau)=\sum_{a=0}^{2 P-1} q^{-\frac{a^{2}}{4 P}} \mathrm{e}^{-2 \pi \mathrm{i} a u} \vartheta_{P, a}(z ; \tau), \\
f_{P}(u, z ; \tau+1)=f_{P}(u, z ; \tau), \\
f_{P}(u, z ; \tau)-\frac{1}{\tau} \mathrm{e}^{2 \pi \mathrm{i} P \frac{u^{2}-z^{2}}{\tau}} f_{P}\left(\frac{u}{\tau}, \frac{z}{\tau} ; \frac{-1}{\tau}\right)=\sum_{a=0}^{2 P-1} M_{P, a}(u ; \tau) \vartheta_{P, a}(z ; \tau),
\end{gathered}
$$

where $\vartheta_{P, a}(z ; \tau)$ are level- $P$ theta functions (A.4), and $M_{P, a}(u ; \tau)$ has a form of the Mordell integral

$$
M_{P, a}(u ; \tau)=\mathrm{i} \mathrm{e}^{-\pi \mathrm{i} \frac{a^{2}}{2 P} \tau-2 \pi \mathrm{i} a u} \int_{\mathbb{R}-\mathrm{i} 0} \frac{\mathrm{e}^{2 \pi \mathrm{i} P \tau x^{2}-2 \pi(2 P u+a \tau) x}}{1-\mathrm{e}^{2 \pi x}} \mathrm{~d} x .
$$

As a completion of $f_{P}(u, z ; \tau)$, we define $\widehat{f}_{P}(u, z ; \tau)$ by

$$
\widehat{f}_{P}(u, z ; \tau)=f_{P}(u, z ; \tau)-\frac{1}{2} \sum_{a} R_{P, a}(u ; \tau) \vartheta_{P, a}(z ; \tau),
$$


where the non-holomorphic partner $R_{P, a}(u ; \tau)$ is given by

$$
\begin{aligned}
& R_{P, a}(u ; \tau) \\
& =\sum_{n \equiv a \in \mathbb{Z}}\left[\operatorname{sgn}\left(n+\frac{1}{2}\right)-E\left(\left(n+2 P \frac{\Im u}{\Im \tau}\right) \sqrt{\frac{\Im \tau}{P}}\right)\right] q^{-\frac{n^{2}}{4 P}} \mathrm{e}^{-2 \pi \mathrm{in} u} .
\end{aligned}
$$

We then find that the function $\widehat{f}_{P}(u, z ; \tau)$ has a modular property similar to a 2-variable Jacobi form with weight 1 ;

$$
\begin{gathered}
\widehat{f}_{P}(u, z ; \tau+1)=\widehat{f}_{P}(u, z ; \tau) \\
\widehat{f}_{P}\left(\frac{u}{\tau}, \frac{z}{\tau} ;-\frac{1}{\tau}\right)=\tau \mathrm{e}^{2 \pi \mathrm{i} P \frac{z^{2}-u^{2}}{\tau}} \widehat{f}_{P}(u, z ; \tau) .
\end{gathered}
$$

4.2. Generalized Mordell Integral. We study the period function of the weight- $3 / 2$ vector-valued modular form $\left\{\Psi_{P}^{(a)}(\tau) \mid 0<a<P\right\}$ defined in (A.9);

$$
M_{P}^{(a)}(\tau)=\int_{0}^{\mathrm{i} \infty} \frac{\Psi_{P}^{(a)}(z)}{\sqrt{\frac{z+\tau}{\mathrm{i}}}} \mathrm{d} z .
$$

We substitute (A.10) for the above integrand, and apply an identity [27, Lemma 1.18]

$$
\int_{-\infty}^{\infty} \frac{\mathrm{e}^{\pi \mathrm{i} \tau w^{2}}}{w+\mathrm{i} r} \mathrm{~d} w=-\pi r \int_{0}^{\mathrm{i} \infty} \frac{\mathrm{e}^{\pi \mathrm{i} r^{2} z}}{\sqrt{\frac{z+\tau}{\mathrm{i}}}} \mathrm{d} z .
$$

Then the period function is rewritten as

$$
M_{P}^{(a)}(\tau)=-\frac{\sqrt{2 P}}{2 \pi} \int_{\mathbb{R}} \mathrm{d} z \mathrm{e}^{\pi \mathrm{i} \tau \frac{z^{2}}{2 P}} \sum_{k \in \mathbb{Z}}\left(\frac{1}{z+\mathrm{i}(2 P k+a)}-\frac{1}{z+\mathrm{i}(2 P k-a)}\right) .
$$

When we make use of an infinite series expansion

$$
\tanh \left(\frac{\pi}{2} x\right)=\frac{4 x}{\pi} \sum_{k=1}^{\infty} \frac{1}{(2 k-1)^{2}+x^{2}}
$$

we find that the period function is written in the form of the generalized Mordell integral as

$$
\begin{aligned}
& M_{P}^{(a)}(\tau) \\
& =-\frac{1}{2 \sqrt{2 P}} \int_{\mathbb{R}} \mathrm{d} z \mathrm{e}^{\pi \mathrm{i} \tau \frac{z^{2}}{2 P}}\left[\tanh \left(\frac{\pi(z+\mathrm{i}(a-P))}{2 P}\right)-\tanh \left(\frac{\pi(z-\mathrm{i}(a-P))}{2 P}\right)\right] \\
& =\frac{\mathrm{i}}{\sqrt{2 P}} \int_{\mathbb{R}} \mathrm{d} z \mathrm{e}^{\pi \mathrm{i} \tau \frac{z^{2}}{2 P}} \frac{\sin \left(\frac{P-a}{P} \pi\right)}{\cosh \left(\frac{z}{P} \pi\right)+\cos \left(\frac{P-a}{P} \pi\right)} .
\end{aligned}
$$

This form of the Mordell integral has also been noted in [23]. These integrals have another form

$$
M_{P}^{(a)}(\tau)=\mathrm{i} \sqrt{\frac{\mathrm{i}}{\tau}} \int_{-\infty}^{\infty} \mathrm{d} x \mathrm{e}^{-\pi \mathrm{i} \frac{x^{2}}{2 P \tau}} \frac{\sinh \left(\pi \frac{P-a}{P} x\right)}{\sinh (\pi x)} .
$$


which is known to generate the Ohtsuki-type invariant for torus link [12]. Note that the generalized Mordell integral (4.11) is also used in studies of the integer partition [2]. Expression (4.9) of the Mordell integral as a period integral easily proves the $S$-transformation formula

$$
\sum_{b=1}^{P-1} \mathbf{S}(P)_{a b} M_{P}^{(b)}(\tau)=\sqrt{\frac{\mathrm{i}}{\tau}} M_{P}^{(a)}\left(-\frac{1}{\tau}\right),
$$

when we substitute (A.11) for (4.9).

Relationship of $M_{P}^{(a)}(\tau)$ with Zwegers' function (4.5) $M_{P, a}(u ; \tau)$ is given as follows: From (4.11), we compute as

$$
\begin{aligned}
M_{P}^{(a)}(\tau) & =\frac{\mathrm{e}^{-\pi \mathrm{i} \tau \frac{(a-P)^{2}}{2 P}}}{2 \sqrt{2 P}} \int_{\mathbb{R}} \mathrm{d} w \tanh \left(\frac{\pi w}{2 P}\right) \mathrm{e}^{\pi \mathrm{i} \tau \frac{w^{2}}{2 P}}\left(\mathrm{e}^{\pi \tau \frac{P-a}{P} w}-\mathrm{e}^{-\pi \tau \frac{P-a}{P} w}\right) \\
& =\frac{\mathrm{e}^{-\pi \mathrm{i} \frac{a^{2}}{2 P} \tau}}{\sqrt{2 P}}\left[\int_{\mathbb{R}+\mathrm{i} 0} \frac{\mathrm{e}^{\pi \mathrm{i} \tau \frac{z^{2}}{2 P}-\pi a \tau \frac{z}{P}}}{1-\mathrm{e}^{-\pi \frac{z}{P}}} \mathrm{~d} z-\int_{\mathbb{R}-\mathrm{i} 0} \frac{\mathrm{e}^{\pi \mathrm{i} \tau \frac{z^{2}}{2 P}-\pi a \tau \frac{z}{P}}}{1-\mathrm{e}^{\pi \frac{z}{P}}} \mathrm{~d} z\right] .
\end{aligned}
$$

Thus we obtain

$$
M_{P}^{(a)}(\tau)=\mathrm{i} \sqrt{2 P}\left[M_{P, a}(0 ; \tau)-M_{P,-a}(0 ; \tau)\right] .
$$

4.3. Non-Holomorphic Function. We define a non-holomorphic function

$$
R_{P}^{(a)}(\tau)=\int_{-\bar{\tau}}^{\mathrm{i} \infty} \frac{\Psi_{P}^{(a)}(z)}{\sqrt{\frac{z+\tau}{\mathrm{i}}}} \mathrm{d} z,
$$

as a generalization of (3.15). This integral expression shows that

$$
\frac{\partial}{\partial \bar{\tau}} R_{P}^{(a)}(\tau)=\frac{\Psi_{P}^{(a)}(-\bar{\tau})}{\sqrt{2 \Im \tau}}
$$

By integrating each summand of $\Psi_{P}^{(a)}(z)$ after substitution of (A.10) into (4.15), we find

$$
R_{P}^{(a)}(\tau)=\mathrm{i} \sqrt{2 P} R_{P, a}(0 ; \tau),
$$

where $R_{P, a}(z ; \tau)$ is Zwegers' $R$-function defined in (4.7).

Substituting the modular transformation law (A.11) for $\Psi_{P}^{(a)}(z)$ in (4.15), we find the transformation law for $R_{P}^{(a)}(\tau)$ as follows;

$$
\begin{gathered}
\sqrt{\frac{\mathrm{i}}{\tau} \sum_{b=1}^{P-1} \mathbf{S}(P)_{a b} R_{P}^{(b)}\left(-\frac{1}{\tau}\right)+R_{P}^{(a)}(\tau)=M_{P}^{(a)}(\tau),} \\
R_{P}^{(a)}(\tau+1)=\mathrm{e}^{-\pi \mathrm{i} \frac{a^{2}}{2 P}} R_{P}^{(a)}(\tau),
\end{gathered}
$$


where $M_{P}^{(a)}(\tau)$ is the Mordell integral defined in (4.9). Using the level- $P \vartheta$-function (A.4), the $S$-transformation formula is rewritten as

$$
\begin{aligned}
& \sum_{a=1}^{P-1} M_{P}^{(a)}(\tau)\left(\vartheta_{P, a}-\vartheta_{P,-a}\right)(z ; \tau)=\left[\sum_{a=1}^{P-1} R_{P}^{(a)}(\tau)\left(\vartheta_{P, a}-\vartheta_{P,-a}\right)(z ; \tau)\right] \\
&-\frac{1}{\tau} \mathrm{e}^{-2 \pi \mathrm{i} \frac{P}{\tau} z^{2}}\left[\sum_{a=1}^{P-1} R_{P}^{(a)}\left(-\frac{1}{\tau}\right)\left(\vartheta_{P, a}-\vartheta_{P,-a}\right)\left(\frac{z}{\tau} ;-\frac{1}{\tau}\right)\right] .
\end{aligned}
$$

4.4. Modular Transformation of Massless Characters. The massless character (4.1) is identified with an anti-symmetric part of the Lerch sum (4.3). Using (4.4) and (4.14), we find

$$
\begin{aligned}
\frac{1}{\mathrm{i} \sqrt{2 P}} \sum_{a=1}^{P-1} M_{P}^{(a)}(\tau)\left[\vartheta_{P, a}(z ; \tau)\right. & \left.-\vartheta_{P,-a}(z ; \tau)\right] \\
= & {\left[\sum_{n \in \mathbb{Z}} q^{P n^{2}} \mathrm{e}^{4 \pi \mathrm{i} P n z} \frac{1+q^{n} \mathrm{e}^{2 \pi \mathrm{i} z}}{1-q^{n} \mathrm{e}^{2 \pi \mathrm{i} z}}\right] } \\
& -\frac{1}{\tau} \mathrm{e}^{-2 \pi \mathrm{i} P \frac{z^{2}}{\tau}}\left[\sum_{n \in \mathbb{Z}} \widetilde{q}^{P n^{2}} \mathrm{e}^{4 \pi \mathrm{i} P n \frac{z}{\tau}} \frac{1+\widetilde{q}^{n} \mathrm{e}^{2 \pi \mathrm{i} \frac{z}{\tau}}}{1-\widetilde{q}^{n} \mathrm{e}^{2 \pi \mathrm{i} \frac{z}{\tau}}}\right],
\end{aligned}
$$

where $\widetilde{q}=\mathrm{e}^{-2 \pi \mathrm{i} / \tau}$. We thus obtain a formula which is a generalization of (3.19);

$$
\begin{aligned}
F_{P}(z ; \tau)+\mathrm{e}^{-2 \pi \mathrm{i}(P-2) \frac{z^{2}}{\tau}} F_{P}\left(\frac{z}{\tau} ;-\frac{1}{\tau}\right) & =\frac{1}{\mathrm{i} \sqrt{2 P}} \sum_{a=1}^{P-1} \frac{M_{P}^{(a)}(\tau)}{\eta(\tau)} \cdot \frac{\vartheta_{P, a}-\vartheta_{P,-a}}{\vartheta_{2,1}-\vartheta_{2,-1}}(z ; \tau) \\
& =\frac{1}{\mathrm{i} \sqrt{2 P}} \sum_{a=1}^{P-1} \frac{M_{P}^{(a)}(\tau)}{\eta(\tau)} \chi_{P-2, \frac{a-1}{2}}(z ; \tau),
\end{aligned}
$$

where we have defined

$$
F_{P}(z ; \tau)=\frac{\mathrm{i}}{\eta(\tau) \theta_{11}(2 z ; \tau)} \sum_{n \in \mathbb{Z}} q^{P n^{2}} \mathrm{e}^{4 \pi \mathrm{i} P n z} \frac{1+q^{n} \mathrm{e}^{2 \pi \mathrm{i} z}}{1-q^{n} \mathrm{e}^{2 \pi \mathrm{i} z}} .
$$

We see that (4.20) with $P=2$ coincides with (3.19), and the massless character (4.1) is related to $F_{P}(z ; \tau)$ as

$$
\operatorname{ch}_{k, \frac{k}{4}, \ell=0}^{\widetilde{R}}(z ; \tau)=\frac{\left[\theta_{11}(z ; \tau)\right]^{2}}{[\eta(\tau)]^{3}} F_{k+1}(z ; \tau)
$$

4.5. Harmonic Maass Form. Looking at the $S$-transformation (4.20) of the massless character, we see that the Mordell integrals can be compensated with those from (4.19). By setting $C_{P}(z ; \tau)$ to be the massless character (4.1) for notational simplicity,

$$
\begin{aligned}
C_{P}(z ; \tau) & =\operatorname{ch}_{k=P-1, h=\frac{P-1}{4}, \ell=0}^{\widetilde{R}}(z ; \tau) \\
& =\frac{\left[\theta_{11}(z ; \tau)\right]^{2}}{[\eta(\tau)]^{3}} \frac{\mathrm{i}}{\theta_{11}(2 z ; \tau)} \sum_{n \in \mathbb{Z}} q^{P n^{2}} \mathrm{e}^{4 \pi \mathrm{i} P n z} \frac{1+q^{n} \mathrm{e}^{2 \pi \mathrm{i} z}}{1-q^{n} \mathrm{e}^{2 \pi \mathrm{i} z}},
\end{aligned}
$$


we introduce a (real analytic) Jacobi as

$$
\widehat{C}_{P}(z ; \tau)=C_{P}(z ; \tau)-\frac{1}{\mathrm{i} \sqrt{2 P}} \sum_{a=1}^{P-1} R_{P}^{(a)}(\tau) B_{P}^{(a)}(z ; \tau)
$$

Here the basis function $B_{P}^{(a)}(z ; \tau)$ is defined as

$$
B_{P}^{(a)}(z ; \tau)=\frac{\left[\theta_{11}(z ; \tau)\right]^{2}}{[\eta(\tau)]^{3}} \cdot \frac{\vartheta_{P, a}-\vartheta_{P,-a}}{\vartheta_{2,1}-\vartheta_{2,-1}}(z ; \tau)=\frac{\left[\theta_{11}(z ; \tau)\right]^{2}}{[\eta(\tau)]^{3}} \chi_{P-2, \frac{a-1}{2}}(z ; \tau) .
$$

The modular transformation formulae of $\widehat{C}_{P}(z ; \tau)$ are summarized as follows;

$$
\begin{gathered}
\widehat{C}_{P}(z ; \tau)=\mathrm{e}^{-2 \pi \mathrm{i}(P-1) \frac{z^{2}}{\tau}} \widehat{C}_{P}\left(\frac{z}{\tau} ;-\frac{1}{\tau}\right), \\
\widehat{C}_{P}(z ; \tau+1)=\widehat{C}_{P}(z+1 ; \tau)=\widehat{C}_{P}(z ; \tau), \\
\widehat{C}_{P}(z+\tau ; \tau)=q^{-(P-1)} \mathrm{e}^{-4 \pi \mathrm{i}(P-1) z} \widehat{C}_{P}(z ; \tau) .
\end{gathered}
$$

We note that the Fourier expansions have the form

$$
\begin{aligned}
& C_{P}(z ; \tau)=1+\left(\mathrm{e}^{\pi \mathrm{i} z}-\mathrm{e}^{-\pi \mathrm{i} z}\right)^{2}\left(\mathrm{e}^{2 \pi \mathrm{i} z}+\mathrm{e}^{-2 \pi \mathrm{i} z}\right) q+\cdots, \\
& B_{P}^{(a)}(z ; \tau)=-\left(\mathrm{e}^{\pi \mathrm{i} z}-\mathrm{e}^{-\pi \mathrm{i} z}\right)^{2} I_{\frac{a-1}{2}}(z) q^{\frac{a^{2}}{4 P}}+\cdots,
\end{aligned}
$$

where $I_{\frac{a}{2}}(z)$ is associated with the Chebyshev polynomial of the second kind;

$$
I_{\frac{a}{2}}(z)=\sum_{n=-\frac{a}{2}}^{\frac{a}{2}} \mathrm{e}^{4 \pi \mathrm{i} n z}
$$

To construct an eigenfunction of the differential operator (3.17) at a higher level, we introduce a holomorphic function

$$
H_{P}^{(a)}(z ; \tau)=\frac{\mathrm{Wr}\left[B_{P}^{(a)} \rightarrow C_{P}\right]}{\mathrm{Wr}\left[B_{P}^{(1)}, \ldots, B_{P}^{(P-1)}\right]}(z ; \tau)
$$

whose completion is given by

$$
\widehat{H}_{P}^{(a)}(z ; \tau)=\frac{\mathrm{Wr}\left[B_{P}^{(a)} \rightarrow \widehat{C}_{P}\right]}{\operatorname{Wr}\left[B_{P}^{(1)}, \ldots, B_{P}^{(P-1)}\right]}(z ; \tau) .
$$

Here $\operatorname{Wr}\left[\phi_{1}, \ldots, \phi_{n}\right](z ; \tau)$ denotes the Wronskian with respect to $z$;

$$
\mathrm{Wr}\left[\phi_{1}, \ldots, \phi_{n}\right](z ; \tau)=\left|\begin{array}{ccc}
\phi_{1}(z ; \tau) & \ldots & \phi_{n}(z ; \tau) \\
\vdots & \ddots & \vdots \\
\frac{\mathrm{d}^{n-1} \phi_{1}}{\mathrm{~d} z^{n-1}}(z ; \tau) & \ldots & \frac{\mathrm{d}^{n-1} \phi_{n}}{\mathrm{~d} z^{n-1}}(z ; \tau)
\end{array}\right|
$$


and $\operatorname{Wr}\left[B_{P}^{(a)} \rightarrow \widehat{C}_{P}\right](z ; \tau)$ means the Wronskian $\operatorname{Wr}\left[B_{P}^{(1)}, \ldots, B_{P}^{(P-1)}\right](z ; \tau)$ with $B_{P}^{(a)}(z ; \tau)$ replaced by $\widehat{C}_{P}(z ; \tau)$. From (4.24) and (4.16), we have

$$
\begin{aligned}
\frac{\partial}{\partial \bar{\tau}} \widehat{H}_{P}^{(a)}(z ; \tau) & =\frac{\mathrm{i}}{\sqrt{2 P}} \cdot \frac{\partial R_{P}^{(a)}(\tau)}{\partial \bar{\tau}} \\
& =\frac{\mathrm{i}}{\sqrt{2 P}} \cdot \frac{\Psi_{P}^{(a)}(-\bar{\tau})}{\sqrt{2 \Im \tau}} .
\end{aligned}
$$

As a result, we have

$$
(\Im \tau)^{\frac{3}{2}} \frac{\partial}{\partial \tau} \sqrt{\Im \tau} \frac{\partial}{\partial \bar{\tau}} \widehat{H}_{P}^{(a)}(z ; \tau)=0
$$

which, with $\tau=u+\mathrm{i} v$, reduces to

$$
\left[-v^{2}\left(\frac{\partial^{2}}{\partial u^{2}}+\frac{\partial^{2}}{\partial v^{2}}\right)+\frac{\mathrm{i} v}{2}\left(\frac{\partial}{\partial u}+\mathrm{i} \frac{\partial}{\partial v}\right)\right] \widehat{H}_{P}^{(a)}(z ; \tau)=0 .
$$

We can check the modular transformation formulae as

$$
\begin{gathered}
\widehat{H}_{P}^{(a)}(z ; \tau)=-\sqrt{\frac{\mathrm{i}}{\tau}} \sum_{b=1}^{P-1} \mathbf{S}(P)_{a b} \widehat{H}_{P}^{(b)}\left(\frac{z}{\tau} ;-\frac{1}{\tau}\right), \\
\widehat{H}_{P}^{(a)}(z ; \tau+1)=\mathrm{e}^{-\frac{a^{2}}{2 P} \pi \mathrm{i}} \widehat{H}_{P}^{(a)}(z ; \tau), \\
\widehat{H}_{P}^{(a)}(z+1 ; \tau)=\widehat{H}_{P}^{(a)}(z+\tau ; \tau)=\widehat{H}_{P}^{(a)}(z ; \tau) .
\end{gathered}
$$

Thus recalling [21], we find that these are the harmonic Maass form with weight $1 / 2$ on $\Gamma(4 P)$.

4.6. Character Decomposition. We define an element of $(P-1) \times(P-1)$ matrix $\mathbf{B}_{P}(\boldsymbol{w} ; \tau)$ by

$$
\left(\mathbf{B}_{P}(\boldsymbol{w} ; \tau)\right)_{a b}=B_{P}^{(b)}\left(w_{a} ; \tau\right),
$$

where $\left\{w_{1}, w_{2}, \ldots, w_{P-1}\right\}$ are coordinates on the torus. As a higher-level generalization of (3.20), we define $J_{P}\left(z ; w_{1}, \ldots, w_{P-1} ; \tau\right)$ by

$$
\begin{aligned}
& J_{P}\left(z ; w_{1}, \ldots, w_{P-1} ; \tau\right) \\
& =\widehat{C}_{P}(z ; \tau)-\sum_{a=1}^{P-1} \sum_{b=1}^{P-1}\left(\mathbf{B}_{P}(\boldsymbol{w} ; \tau)^{-1}\right)_{a b} \widehat{C}_{P}\left(w_{b} ; \tau\right) B_{P}^{(a)}(z ; \tau) \\
& =C_{P}(z ; \tau)-\sum_{a=1}^{P-1} \sum_{b=1}^{P-1}\left(\mathbf{B}_{P}(\boldsymbol{w} ; \tau)^{-1}\right)_{a b} C_{P}\left(w_{b} ; \tau\right) B_{P}^{(a)}(z ; \tau),
\end{aligned}
$$

where the second equality follows from the $z$-independence of the function $R_{P}^{(a)}(\tau)$ (4.24). The function $J_{P}\left(z ; w_{1}, \ldots, w_{P-1} ; \tau\right)$ has a holomorphic $q$-series as seen from the second 
equality, and it has a good behavior under modular transformations. Explicitly we have

$$
\begin{aligned}
& J_{P}\left(z ; w_{1}, \ldots, w_{P-1} ; \tau\right)=\mathrm{e}^{-2 \pi \mathrm{i}(P-1) \frac{z^{2}}{\tau}} J_{P}\left(\frac{z}{\tau} ; \frac{w_{1}}{\tau}, \ldots, \frac{w_{P-1}}{\tau} ;-\frac{1}{\tau}\right) \\
& J_{P}\left(z+1 ; w_{1}, \ldots, w_{P-1} ; \tau\right)=J_{P}\left(z ; w_{1}, \ldots, w_{a}+1, \ldots, w_{P-1} ; \tau\right) \\
&=J_{P}\left(z ; w_{1}, \ldots, w_{a}+\tau, \ldots, w_{P-1} ; \tau\right) \\
&=J_{P}\left(z ; w_{1}, \ldots, w_{P-1} ; \tau+1\right) \\
&=J_{P}\left(z ; w_{1}, \ldots, w_{P-1} ; \tau\right) \\
& J_{P}\left(z+\tau ; w_{1}, \ldots, w_{P-1} ; \tau\right)= q^{-(P-1)} \mathrm{e}^{-4 \pi \mathrm{i}(P-1) z} J_{P}\left(z ; w_{1}, \ldots, w_{P-1} ; \tau\right) .
\end{aligned}
$$

By construction, the function $J_{P}\left(z ; w_{1}, \ldots, w_{P-1} ; \tau\right)$ vanishes at $z=w_{a}$ for $a=1, \ldots, P-1$,

$$
J_{P}\left(w_{a} ; w_{1}, \ldots, w_{P-1} ; \tau\right)=0
$$

and we also have

$$
J_{P}\left(0 ; w_{1}, \ldots, w_{P-1} ; \tau\right)=1
$$

4.6.1. Degenerate Case. We consider the completely degenerate configuration, $w_{1}=w_{2}=$ $\cdots=w_{P-1}$; we set

$$
J_{P}(z ; w ; \tau)=\lim _{\substack{w_{a} \rightarrow w \\ \forall a}} J_{P}\left(z ; w_{1}, \ldots, w_{P-1} ; \tau\right) .
$$

We then obtain from (4.34)

$$
\begin{aligned}
J_{P}(z ; w ; \tau) & =\widehat{C}_{P}(z ; \tau)-\sum_{a=1}^{P-1} \widehat{H}_{P}^{(a)}(w ; \tau) B_{P}^{(a)}(z ; \tau) \\
& =C_{P}(z ; \tau)-\sum_{a=1}^{P-1} H_{P}^{(a)}(w ; \tau) B_{P}^{(a)}(z ; \tau),
\end{aligned}
$$

where $\widehat{H}_{P}^{(a)}(z ; \tau)$ is the harmonic Maass form of (4.29), and $H_{P}^{(a)}(z ; \tau)$ is its holomorphic part (4.28). The second line above gives a decomposition of a massless character $C_{P}(z ; \tau)$ into a Jacobi form $J_{P}(z ; w ; \tau)$ and a sum over basis functions $B_{P}^{(a)}(z ; \tau)$. Note that $J_{P}(z ; w ; \tau)$ has $2(P-1)$-th order zero at $z=w$. Its transformation formulae are given as

$$
\begin{aligned}
J_{P}(z ; w ; \tau)= & \mathrm{e}^{-2 \pi \mathrm{i}(P-1) \frac{z^{2}}{\tau}} J_{P}\left(\frac{z}{\tau} ; \frac{w}{\tau} ; \frac{-1}{\tau}\right), \\
J_{P}(z+1 ; w ; \tau) & =J_{P}(z ; w+1 ; \tau) \\
& =J_{P}(z ; w+\tau ; \tau) \\
& =J_{P}(z ; w ; \tau+1)=J_{P}(z ; w ; \tau), \\
J_{P}(z+\tau ; w ; \tau) & =q^{-(P-1)} \mathrm{e}^{-4 \pi \mathrm{i}(P-1) z} J_{P}(z ; w ; \tau) .
\end{aligned}
$$


When we set $w$ to special values $\left\{\frac{1}{2}, \frac{1+\tau}{2}, \frac{\tau}{2}\right\}$, these formulas give the following identifications;

$J_{P}\left(z ; \frac{1}{2} ; \tau\right)=\left(\frac{\theta_{10}(z ; \tau)}{\theta_{10}(0 ; \tau)}\right)^{2(P-1)}, \quad J_{P}\left(z ; \frac{1+\tau}{2} ; \tau\right)=\left(\frac{\theta_{00}(z ; \tau)}{\theta_{00}(0 ; \tau)}\right)^{2(P-1)} \quad J_{P}\left(z ; \frac{\tau}{2} ; \tau\right)=\left(\frac{\theta_{01}(z ; \tau)}{\theta_{01}(0 ; \tau)}\right)^{2(P-1)}$

Hence we have

$$
\begin{aligned}
\operatorname{ch}_{k=P-1, h=\frac{P-1}{4}, \ell=0}^{\widetilde{R}}(z ; \tau) & =\left(\frac{\theta_{10}(z ; \tau)}{\theta_{10}(0 ; \tau)}\right)^{2(P-1)}+\sum_{a=1}^{P-1} H_{P}^{(a)}\left(\frac{1}{2} ; \tau\right) B_{P}^{(a)}(z ; \tau) \\
& =\left(\frac{\theta_{00}(z ; \tau)}{\theta_{00}(0 ; \tau)}\right)^{2(P-1)}+\sum_{a=1}^{P-1} H_{P}^{(a)}\left(\frac{1+\tau}{2} ; \tau\right) B_{P}^{(a)}(z ; \tau) \\
& =\left(\frac{\theta_{01}(z ; \tau)}{\theta_{01}(0 ; \tau)}\right)^{2(P-1)}+\sum_{a=1}^{P-1} H_{P}^{(a)}\left(\frac{\tau}{2} ; \tau\right) B_{P}^{(a)}(z ; \tau)
\end{aligned}
$$

4.7. Elliptic Genera. Character decomposition above can be used to rewrite the elliptic genera of hyper-Kähler manifolds $X_{k}$ with $\operatorname{dim}_{\mathbb{C}} X_{k}=2 k, k \geq 1$.

We note that the elliptic genera for hyper-Kähler manifolds $X_{k}$ is in general given by a sum of symmetric polynomials of $\left\{\left(\frac{\theta_{10}(z ; \tau)}{\theta_{10}(0 ; \tau)}\right)^{2},\left(\frac{\theta_{00}(z ; \tau)}{\theta_{00}(0 ; \tau)}\right)^{2},\left(\frac{\theta_{01}(z ; \tau)}{\theta_{01}(0 ; \tau)}\right)^{2}\right\}$ with order $k$. In the case of a polynomial of the following form

$$
Z_{X_{k}}^{(1)}(z ; \tau)=c_{k} 2^{2 k}\left[\left(\frac{\theta_{10}(z ; \tau)}{\theta_{10}(0 ; \tau)}\right)^{2 k}+\left(\frac{\theta_{00}(z ; \tau)}{\theta_{00}(0 ; \tau)}\right)^{2 k}+\left(\frac{\theta_{01}(z ; \tau)}{\theta_{01}(0 ; \tau)}\right)^{2 k}\right],
$$

we can use the character decomposition of the completely degenerate configuration (4.44), (4.45), (4.46). Here we introduced a factor $2^{2 k}$ for convenience and the parameter $c_{k} \in \mathbb{Z}_{>0}$ is to be fixed later so that $Z_{X_{k}}^{(1)}$ contains the identity representation with multiplicity 1 in the NS sector.

In the case of a polynomial of the form

$$
\left[\left(\frac{\theta_{10}(z ; \tau)}{\theta_{10}(0 ; \tau)}\right)^{2 k_{2}}\left(\frac{\theta_{00}(z ; \tau)}{\theta_{00}(0 ; \tau)}\right)^{2 k_{3}}\left(\frac{\theta_{01}(z ; \tau)}{\theta_{01}(0 ; \tau)}\right)^{2 k_{4}}+\text { permutations }\right]
$$

one can use the character decomposition (4.35) corresponding to the choice of parameters $w_{1}, \cdots, w_{P-1}$
$\boldsymbol{w}_{k_{2}, k_{3}, k_{4}}=\left\{\begin{array}{c}w_{1}, \ldots, w_{k} \mid \quad \#\left(w_{a}=\frac{1}{2}\right)=k_{2}, \#\left(w_{a}=\frac{1+\tau}{2}\right)=k_{3}, \#\left(w_{a}=\frac{\tau}{2}\right)=k_{4}, \\ k_{2}+k_{3}+k_{4}=k .\end{array}\right\}$.

In fact the Jacobi form corresponding to the vector $\boldsymbol{w}_{k_{2}, k_{3}, k_{4}}$ is given by

$$
J_{k+1}\left(z ; \boldsymbol{w}_{k_{2}, k_{3}, k_{4}} ; \tau\right)=\left(\frac{\theta_{10}(z ; \tau)}{\theta_{10}(0 ; \tau)}\right)^{2 k_{2}}\left(\frac{\theta_{00}(z ; \tau)}{\theta_{00}(0 ; \tau)}\right)^{2 k_{3}}\left(\frac{\theta_{01}(z ; \tau)}{\theta_{01}(0 ; \tau)}\right)^{2 k_{4}}
$$


Let us first consider the completely degenerate case. Using (4.44)-(4.46) and (4.25), we obtain

$$
\begin{aligned}
c_{k}^{-1} 2^{-2 k} Z_{X_{k}}^{(1)}(z ; \tau)=3 \operatorname{ch}_{k, h=\frac{k}{4}, \ell=0}^{\widetilde{R}}(z ; \tau) & \\
& -\sum_{a=1}^{k} \sum_{w \in\left\{\frac{1}{2}, \frac{1+\tau}{2}, \frac{\tau}{2}\right\}} H_{k+1}^{(a)}(w ; \tau) \frac{\left[\theta_{11}(z ; \tau)\right]^{2}}{[\eta(\tau)]^{3}} \chi_{k-1, \frac{a-1}{2}}(z ; \tau) .
\end{aligned}
$$

It turns out that the coefficients of lowest powers of the Fourier expansion of $H_{P}^{(a)}(w ; \tau)$ with $\tau \in\left\{\frac{1}{2}, \frac{1+\tau}{2}, \frac{\tau}{2}\right\}$ are conveniently computed from the expansion of Jacobi forms (4.43). Namely by use of (4.27) and (A.2) with a help of an identity of the Chebyshev polynomial

$$
\left(\mathrm{e}^{\pi \mathrm{i} z}-\mathrm{e}^{-\pi \mathrm{i} z}\right)^{2 n}=(-1)^{n} \sum_{k=0}^{n}(-1)^{k} \frac{2(k+1)}{n+k+2}\left(\begin{array}{c}
2 n+1 \\
n-k
\end{array}\right) I_{\frac{k}{2}}(z),
$$

we find that

$$
\begin{aligned}
H_{P}^{(a)}\left(\frac{1}{2} ; \tau\right) & =q^{-\frac{a^{2}}{4 P}}\left(\alpha_{P}^{(a)}+\mathcal{O}(q)\right) \\
H_{P}^{(a)}\left(\frac{1+\tau}{2} ; \tau\right) & =q^{-\frac{a^{2}}{4 P}+\frac{a}{2}}\left(\beta_{P-1, P-1-a}+\mathcal{O}\left(q^{\frac{1}{2}}\right)\right) \\
H_{P}^{(a)}\left(\frac{\tau}{2} ; \tau\right) & =q^{-\frac{a^{2}}{4 P}+\frac{a}{2}}\left((-1)^{a} \cdot \beta_{P-1, P-1-a}+\mathcal{O}\left(q^{\frac{1}{2}}\right)\right) .
\end{aligned}
$$

Here we have defined $\alpha_{P}^{(a)}(a=1,2, \ldots, P-1)$ and $\beta_{n, k}$ by

$$
\begin{gathered}
\alpha_{P}^{(a)}=\sum_{m=a}^{P-1} \frac{(-1)^{m+a}}{2^{2 m-1}} \frac{a}{m+a}\left(\begin{array}{c}
P-1 \\
m
\end{array}\right)\left(\begin{array}{c}
2 m-1 \\
m-a
\end{array}\right), \\
\beta_{n, k}=\frac{2(k+1)}{n+k+2}\left(\begin{array}{c}
2 n+1 \\
n-k
\end{array}\right) .
\end{gathered}
$$

These results agree with Fourier coefficients of $H_{P}^{(a)}(z ; \tau)$ computed directly from (4.28) in Appendix B.

As we see from the Appendix, the $q$-expansion of $H_{P}^{(a)}\left(\frac{1}{2} ; \tau\right)$ is always with integer powers of $q$ (upto an overall factor $\left.q^{-\frac{a^{2}}{4 P}}\right)$ while the sum of $H_{P}^{(a)}\left(\frac{1+\tau}{2} ; \tau\right)$ and $H_{P}^{(a)}\left(\frac{\tau}{2} ; \tau\right)$ has either all integer powers (for $a=$ even) or half-integer powers (for $a=$ odd) up to an overall factor $q^{-\frac{a^{2}}{4 P}+\frac{a}{2}}$. Sum of $H_{P}^{(a)}$-functions have the $q$-expansion of the form

$$
H_{P}^{(a)}\left(\frac{1}{2} ; \tau\right)+H_{P}^{(a)}\left(\frac{1+\tau}{2} ; \tau\right)+H_{P}^{(a)}\left(\frac{\tau}{2} ; \tau\right)=q^{-\frac{a^{2}}{4(k+1)}}\left[\alpha_{k+1}^{(a)}-\sum_{n \geq 1} A_{k+1, n}^{(a)} q^{n}\right]
$$


Substituting above into $Z_{X_{k}}^{(1)}$, we find

$$
\begin{aligned}
c_{k}^{-1} 2^{-2 k} Z_{X_{k}}^{(1)}(z ; \tau) & =3 \operatorname{ch}_{k, h=\frac{k}{4}, \ell=0}^{\widetilde{R}}(z ; \tau) \\
& -\sum_{a=1}^{k} q^{-\frac{a^{2}}{4(k+1)}}\left[\alpha_{k+1}^{(a)}-\sum_{n \geq 1} A_{k+1, n}^{(a)} q^{n}\right] \frac{\left[\theta_{11}(z ; \tau)\right]^{2}}{[\eta(\tau)]^{3}} \chi_{k-1, \frac{a-1}{2}}(z ; \tau) .
\end{aligned}
$$

Note that while terms with the coefficients $A_{k+1, n}^{(a)}$ give massive representations with $h=$ $n+k / 4$, terms with coefficients $\alpha_{k+1}^{(a)}$ are a sum of massless representations because of the relation (4.2).

We then rewrite (4.51) using (4.2) and find

$$
\frac{1}{c_{k}} Z_{X_{k}}^{(1)}(z ; \tau)=\sum_{a=0}^{k}(-1)^{a} \gamma_{k, a} \operatorname{ch}_{k, \frac{k}{4}, \frac{a}{2}}^{\widetilde{R}}(z ; \tau)+\text { massive characters. }
$$

Here a coefficient $\gamma_{k, a}$ of isospin- $a / 2$ massless representation is given by

$$
\gamma_{k, a}=2^{2 k}\left(\alpha_{k+1}^{(a)}-2 \alpha_{k+1}^{(a+1)}+\alpha_{k+1}^{(a+2)}\right)
$$

where in our convention we have

$$
\alpha_{k+1}^{(0)}=3 .
$$

As we see from Table 1 we have $\gamma_{k, k}=1$, which counts the multiplicity of massless representations with isospin $\frac{k}{2}$ in the $R$-sector. Since the spectral flow (in the left-moving sector) sends this representation to identity representation in the NS sector while the right-moving sector gives a degeneracy of $k+1$ for spin $k / 2$ representation, we should multiply $Z_{X_{k}}^{(1)}$ by an overall factor $(k+1)$ : then the multiplicity of identity representation in NS sector is adjusted to 1 . Thus the normalization parameter in (4.47) fixes to

$$
c_{k}=k+1 \text {. }
$$

We see that (4.53) is computed for $a>0$ as

$$
\begin{aligned}
\gamma_{k, a} & =2^{2 k} \sum_{m=a}^{k} \frac{(-1)^{m+a}}{2^{2 m-1}} \frac{a+1}{m+a+2}\left(\begin{array}{c}
k \\
m
\end{array}\right)\left(\begin{array}{c}
2 m+1 \\
m-a
\end{array}\right) \\
& =\frac{2(a+1)}{k+a+2}\left(\begin{array}{c}
2 k+1 \\
k-a
\end{array}\right),
\end{aligned}
$$

and that

$$
\gamma_{k, 0}=C_{k+1}+2^{2 k+1},
$$

with the Catalan number $C_{n}=\frac{1}{n+1}\left(\begin{array}{c}2 n \\ n\end{array}\right)$. We then have

$$
\frac{k+1}{a+1} \gamma_{k, a} \in \mathbb{Z}
$$

which gives the net number of massless representations with spin $\frac{k-a}{2}$ in the NS sector. See Table 2. 
SUPERCONFORMAL ALGEBRAS AND MOCK THETA FUNCTIONS

\begin{tabular}{|c|c|c|c|c|c|c|c|c|c|c|c|}
\hline$k \backslash a$ & 0 & 1 & 2 & 3 & 4 & 5 & 6 & 7 & 8 & 9 & 10 \\
\hline 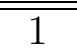 & 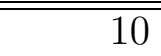 & 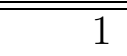 & & & & & & & & & \\
\hline 2 & 37 & 4 & 1 & & & & & & & & \\
\hline 3 & 142 & 14 & 6 & 1 & & & & & & & \\
\hline 4 & 554 & 48 & 27 & 8 & 1 & & & & & & \\
\hline 5 & 2180 & 165 & 110 & 44 & 10 & 1 & & & & & \\
\hline 6 & 8621 & 572 & 429 & 208 & 65 & 12 & 1 & & & & \\
\hline 7 & 34198 & 2002 & 1638 & 910 & 350 & 90 & 14 & 1 & & & \\
\hline 8 & 135934 & 7072 & 6188 & 3808 & 1700 & 544 & 119 & 16 & 1 & & \\
\hline 9 & 541084 & 25194 & 23256 & 15504 & 7752 & 2907 & 798 & 152 & 18 & 1 & \\
\hline 10 & 2155938 & 90440 & 87210 & 62016 & 33915 & 14364 & 4655 & 1120 & 189 & 20 & 1 \\
\hline
\end{tabular}

\begin{tabular}{|c|c|c|c|c|c|c|c|c|c|c|c|}
\hline$k \backslash a$ & 0 & 1 & 2 & 3 & 4 & 5 & 6 & 7 & 8 & 9 & 10 \\
\hline 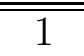 & $\overline{20}$ & 1 & & & & & & & & & \\
\hline 2 & 111 & 6 & 1 & & & & & & & & \\
\hline 3 & 568 & 28 & 8 & 1 & & & & & & & \\
\hline 4 & 2770 & 120 & 45 & 10 & 1 & & & & & & \\
\hline 5 & 13080 & 495 & 220 & 66 & 12 & 1 & & & & & \\
\hline 6 & 60347 & 2002 & 1001 & 364 & 91 & 14 & 1 & & & & \\
\hline 7 & 273584 & 8008 & 4368 & 1820 & 560 & 120 & 16 & 1 & & & \\
\hline 8 & 1223406 & 31824 & 18564 & 8568 & 3060 & 816 & 153 & 18 & 1 & & \\
\hline 9 & 5410840 & 125970 & 77520 & 38760 & 15504 & 4845 & 1140 & 190 & 20 & 1 & \\
\hline 10 & 23715318 & 497420 & 319770 & 170544 & 74613 & 26334 & 7315 & 1540 & 231 & 22 & 1 \\
\hline
\end{tabular}

TABLE 2. $\frac{k+1}{a+1} \gamma_{k, a}$ for $a=0,1,2, \ldots, k$.

We note that a series $\gamma_{k, a}$ for $a=1,2, \ldots, k$ has a combinatorial interpretation [22, A050156]; we consider a set of sequences of 0's and 1's,

$$
V(k)=\left\{v_{1} v_{2} \cdots v_{2 k+1} \mid \#(1 ' s)=k+2, \#(0 ' s)=k-1\right\} .
$$

For each element $v_{1} \cdots v_{2 k+1} \in V(k)$, we have a subsequence $v_{1} v_{2} \cdots v_{h}$ for $h=1,2, \ldots, 2 k+$ 1. Then we observe that $\gamma_{k, a}$ denotes a cardinality of a subset $V(k)$ such that

$$
\max _{h}\{\#(1 ' s)-\#(0 ' s)\}=a+2 .
$$

From a viewpoint of statistical mechanics, a set $V(k)$ of sequences is regarded as a staircase walk which starts from $(0,0)$ and ends at $(k-1, k+2)$. Here 0 (resp. 1$)$ corresponds to a unit right-walk $\rightarrow$ (resp. up-walk $\uparrow$ ). Then $\gamma_{k, a}$ coincides with the number of staircase walks which touch a line $y=x+a+2$ and do not go beyond the line.

4.7.1. Other Cases. Along the same strategy as the completely degenerate case, we can decompose symmetric polynomials of the Jacobi theta functions by using a suitable choice of points $w_{1} \cdots, w_{P-1}$.

Upon symmetrization, we obtain the following; 
(a) $k_{2}>k_{3}>k_{4}$

$$
\begin{aligned}
2^{2 k_{2}-1} & \left(\frac{\theta_{10}(z ; \tau)}{\theta_{10}(0 ; \tau)}\right)^{2 k_{2}}\left(\frac{\theta_{00}(z ; \tau)}{\theta_{00}(0 ; \tau)}\right)^{2 k_{3}}\left(\frac{\theta_{01}(z ; \tau)}{\theta_{01}(0 ; \tau)}\right)^{2 k_{4}}+\text { other } 5 \text { terms } \\
= & \sum_{a=0}^{k_{2}}(-1)^{a}\left(\gamma_{k_{2}, a}+2^{2 k_{2}-2 k_{3}} \gamma_{k_{3}, a}+2^{2 k_{2}-2 k_{4}} \gamma_{k_{4}, a}\right) \operatorname{ch}_{k, \frac{k}{4}, \frac{a}{2}}^{\widetilde{R}}(z ; \tau)+\text { massive characters, }
\end{aligned}
$$

(b) $k_{2}=k_{3} \neq k_{4}$

$$
\begin{aligned}
2^{2 k_{2}-1} & \left(\frac{\theta_{10}(z ; \tau)}{\theta_{10}(0 ; \tau)} \cdot \frac{\theta_{00}(z ; \tau)}{\theta_{00}(0 ; \tau)}\right)^{2 k_{2}}\left(\frac{\theta_{01}(z ; \tau)}{\theta_{01}(0 ; \tau)}\right)^{2 k_{4}}+\text { other } 2 \text { terms } \\
= & \sum_{a=0}^{\max \left(k_{2}, k_{4}\right)}(-1)^{a}\left(\gamma_{k_{2}, a}+2^{2 k_{2}-2 k_{4}-1} \gamma_{k_{4}, a}\right) \operatorname{ch}_{k, \frac{k}{4}, \frac{a}{2}}^{\widetilde{R}}(z ; \tau)+\text { massive characters, }
\end{aligned}
$$

(c) $k_{2}=k_{3}=k_{4}$,

$$
\begin{aligned}
& 2^{2 k_{2}}\left(\frac{\theta_{10}(z ; \tau)}{\theta_{10}(0 ; \tau)} \cdot \frac{\theta_{00}(z ; \tau)}{\theta_{00}(0 ; \tau)} \cdot \frac{\theta_{01}(z ; \tau)}{\theta_{01}(0 ; \tau)}\right)^{2 k_{2}} \\
& =\sum_{a=0}^{k_{2}}(-1)^{a} \gamma_{k_{2}, a} \operatorname{ch}_{k, \frac{k}{4}, \frac{a}{2}}^{\widetilde{R}}(z ; \tau)+\text { massive characters. }
\end{aligned}
$$

Here $\gamma_{k, a}$ is defined by (4.53) with

$$
\alpha_{k+1}^{(0)}=1,
$$

which coincides with (4.55) for $a \geq 0$. Normalization factors in the left hand sides in (4.58) are fixed so that coefficients of the massless $\operatorname{character} \operatorname{ch}_{k, \frac{k}{4}, \frac{a}{2}}(z ; \tau)$ are integral. We emphasize that the massless isospin $k / 2$ representation appears only in the completely degenerate case $\left(k_{2}, k_{3}, k_{4}\right)=(k, 0,0)$ for which the overall normalization can be fixed by requiring the multiplicity to be one for the vacuum representation in NS sector. We cannot fix the overall normalization in the case of other configurations.

4.8. Example: Hyper-Kähler Manifold with $\operatorname{dim}_{\mathbb{C}}=4$. We have two cases, $\left(k_{2}, k_{3}, k_{4}\right)=(2,0,0)$ and $(1,1,0)$. The former case is (4.47) with $k=2$;

$$
\begin{aligned}
48\left[\left(\frac{\theta_{10}(z ; \tau)}{\theta_{10}(0 ; \tau)}\right)^{4}+\right. & \left.\left(\frac{\theta_{00}(z ; \tau)}{\theta_{00}(0 ; \tau)}\right)^{4}+\left(\frac{\theta_{01}(z ; \tau)}{\theta_{01}(0 ; \tau)}\right)^{4}\right] \\
= & 111 \operatorname{ch}_{2, \frac{2}{4}, 0}^{\widetilde{R}}(z ; \tau)-12 \operatorname{ch}_{2, \frac{2}{4}, \frac{1}{2}}^{\widetilde{R}}(z ; \tau)+3 \operatorname{ch}_{2, \frac{2}{4}, 1}^{\widetilde{R}}(z ; \tau) \\
+(1872 q+ & \left.26070 q^{2}+213456 q^{3}+1311420 q^{4}+\cdots\right) q^{-\frac{1}{12}} B_{3}^{(1)}(z ; \tau) \\
& -\left(510 q+12804 q^{2}+126360 q^{3}+841176 q^{4}+\cdots\right) q^{-\frac{1}{3}} B_{3}^{(2)}(z ; \tau) .
\end{aligned}
$$


The latter case follows from (4.58b) and reads as

$$
\begin{aligned}
& 2\left[\left(\frac{\theta_{10}(z ; \tau)}{\theta_{10}(0 ; \tau)} \cdot \frac{\theta_{00}(z ; \tau)}{\theta_{00}(0 ; \tau)}\right)^{2}+\left(\frac{\theta_{00}(z ; \tau)}{\theta_{00}(0 ; \tau)} \cdot \frac{\theta_{01}(z ; \tau)}{\theta_{01}(0 ; \tau)}\right)^{2}+\left(\frac{\theta_{01}(z ; \tau)}{\theta_{01}(0 ; \tau)} \cdot \frac{\theta_{10}(z ; \tau)}{\theta_{10}(0 ; \tau)}\right)^{2}\right] \\
&=4 \operatorname{ch}_{2, \frac{2}{4}, 0}^{\widetilde{R}}(z ; \tau)-\operatorname{ch}_{2, \frac{2}{4}, \frac{1}{2}}^{\widetilde{R}}(z ; \tau)+\left(16 q+55 q^{2}+144 q^{3}+330 q^{4} \cdots\right) q^{-\frac{1}{12}} B_{3}^{(1)}(z ; \tau) \\
&+\left(10 q+44 q^{2}+110 q^{3}+280 q^{4}+\cdots\right) q^{-\frac{1}{3}} B_{3}^{(2)}(z ; \tau) .
\end{aligned}
$$

In the above decomposition the isospin 1/2 massless representation has a coefficient 1 : we should multiply an overall factor 2 to account for the degeneracy of the right-moving sector. Furthermore we require that all coefficients of massless and massive characters should be positive integers in the NS sector when we combine the above 2 cases. Then the elliptic genus of hyper-Kähler manifold $X_{2}(n)$ with complex dimension 4 may be written as

$$
\begin{aligned}
& Z_{X_{2}(n)}(z ; \tau)=48\left[\left(\frac{\theta_{10}(z ; \tau)}{\theta_{10}(0 ; \tau)}\right)^{4}+\left(\frac{\theta_{00}(z ; \tau)}{\theta_{00}(0 ; \tau)}\right)^{4}+\left(\frac{\theta_{01}(z ; \tau)}{\theta_{01}(0 ; \tau)}\right)^{4}\right] \\
& \quad+4 n\left[\left(\frac{\theta_{10}(z ; \tau)}{\theta_{10}(0 ; \tau)} \cdot \frac{\theta_{00}(z ; \tau)}{\theta_{00}(0 ; \tau)}\right)^{2}+\left(\frac{\theta_{00}(z ; \tau)}{\theta_{00}(0 ; \tau)} \cdot \frac{\theta_{01}(z ; \tau)}{\theta_{01}(0 ; \tau)}\right)^{2}+\left(\frac{\theta_{01}(z ; \tau)}{\theta_{01}(0 ; \tau)} \cdot \frac{\theta_{10}(z ; \tau)}{\theta_{10}(0 ; \tau)}\right)^{2}\right],
\end{aligned}
$$

where $n$ is an integer satisfying

$$
-6 \leq n \leq 25
$$

We have

$$
\begin{aligned}
Z_{X_{2}(n)}(z=0 ; \tau) & =12(n+12), \\
Z_{X_{2}(n)}\left(z=\frac{1}{2} ; \tau\right) & =4(n+24)+12288 q+294912 q^{2}+\cdots, \\
Z_{X_{2}(n)}\left(z=\frac{1+\tau}{2} ; \tau\right) & =3 q^{-1}-4(n+6) q^{-\frac{1}{2}}+828+\cdots,
\end{aligned}
$$

In the mathematical literature, see for instance [19], two representative examples of complex 4-dimensional hyper-Kähler manifolds are discussed: Hilbert scheme of points on K3 surfaces $K^{[2]}$ and complex tori $A^{[3]]}$. Their topological invariants are given by

\begin{tabular}{c|ccc}
$X$ & $\chi_{X}$ & $\sigma_{X}$ & $\widehat{A}_{X}$ \\
\hline \hline$K^{[2]}$ & 324 & 156 & 3 \\
$A^{[3]]}$ & 108 & 84 & 3
\end{tabular}

These values agree exactly with those of (4.60) for $n=15\left(K^{[2]}\right)$ and $n=-3\left(A^{[[3]]}\right)$, respectively. We in particular predict $\widehat{A}=3$ for any hyper-Kähler manifold in 4-dimensions.

\section{Concluding Remarks}

We have developed a method of improving the modular properties of BPS representations in $\mathcal{N}=4$ SCA by taking an analogy with a theory of mock theta functions. We have 
obtained a systematic treatment of character decomposition which can be applied to study the elliptic genera of hyper-Kähler manifolds. Our treatment of positivity and integrality properties on the infinite series of massive representations is yet incomplete. We would like to discuss these issues in detail and also the case of higher dimensional hyper-Kähler manifolds in forthcoming publications.

\section{ACKNOWLEDGMENTS}

T.E. would like to thank Y. Sugawara and A. Taormina for discussions and introduction to mathematical literature on mock theta functions. K.H. would like to thank K. Bringmann and J. Lovejoy for useful communications. This work is supported in part by Grant-in-Aid from the Ministry of Education, Culture, Sports, Science and Technology of Japan.

\section{Appendix A. Theta Functions}

A.1. Jacobi Theta Function. The Jacobi theta functions are defined by

$$
\begin{aligned}
& \theta_{11}(z ; \tau)=\sum_{n \in \mathbb{Z}} q^{\frac{1}{2}\left(n+\frac{1}{2}\right)^{2}} \mathrm{e}^{2 \pi \mathrm{i}\left(n+\frac{1}{2}\right)\left(z+\frac{1}{2}\right)}=-\mathrm{i} \theta_{1}(z ; \tau), \\
& \theta_{10}(z ; \tau)=\sum_{n \in \mathbb{Z}} q^{\frac{1}{2}\left(n+\frac{1}{2}\right)^{2}} \mathrm{e}^{2 \pi \mathrm{i}\left(n+\frac{1}{2}\right) z}=\theta_{2}(z ; \tau), \\
& \theta_{00}(z ; \tau)=\sum_{n \in \mathbb{Z}} q^{\frac{1}{2} n^{2}} \mathrm{e}^{2 \pi \mathrm{i} n z}=\theta_{3}(z ; \tau), \\
& \theta_{01}(z ; \tau)=\sum_{n \in \mathbb{Z}} q^{\frac{1}{2} n^{2}} \mathrm{e}^{2 \pi \mathrm{i} n\left(z+\frac{1}{2}\right)}=\theta_{4}(z ; \tau),
\end{aligned}
$$

where we have also shown the relation to the conventional notations. Under the $S$ transformation, we have

$$
\left(\begin{array}{c}
\theta_{11}(z ; \tau) \\
\theta_{10}(z ; \tau) \\
\theta_{00}(z ; \tau) \\
\theta_{01}(z ; \tau)
\end{array}\right)=\sqrt{\frac{\mathrm{i}}{\tau}} \mathrm{e}^{-\pi \mathrm{i} \frac{z^{2}}{\tau}}\left(\begin{array}{llll}
\mathrm{i} & & & \\
& & & 1 \\
& & 1 & \\
& 1 & &
\end{array}\right)\left(\begin{array}{c}
\theta_{11}\left(\frac{z}{\tau} ;-\frac{1}{\tau}\right) \\
\theta_{10}\left(\frac{z}{\tau} ;-\frac{1}{\tau}\right) \\
\theta_{00}\left(\frac{z}{\tau} ;-\frac{1}{\tau}\right) \\
\theta_{01}\left(\frac{z}{\tau} ;-\frac{1}{\tau}\right)
\end{array}\right) .
$$

We see that for $k \in \mathbb{Z}$

$$
\begin{gathered}
\left(\frac{\theta_{10}(z ; \tau)}{\theta_{10}(0 ; \tau)}\right)^{2 k}=\left(\frac{\mathrm{e}^{\pi \mathrm{i} z}+\mathrm{e}^{-\pi \mathrm{i} z}}{2}\right)^{2 k}\left(1+2 k\left(\mathrm{e}^{\pi \mathrm{i} z}-\mathrm{e}^{-\pi \mathrm{i} z}\right)^{2} q+\cdots\right) \\
\left(\frac{\theta_{00}(z ; \tau)}{\theta_{00}(0 ; \tau)}\right)^{2 k}=1+2 k\left(\mathrm{e}^{\pi \mathrm{i} z}-\mathrm{e}^{-\pi \mathrm{i} z}\right)^{2} q^{\frac{1}{2}}+\cdots \\
\left(\frac{\theta_{01}(z ; \tau)}{\theta_{01}(0 ; \tau)}\right)^{2 k}=1-2 k\left(\mathrm{e}^{\pi \mathrm{i} z}-\mathrm{e}^{-\pi \mathrm{i} z}\right)^{2} q^{\frac{1}{2}}+\cdots
\end{gathered}
$$


A.2. Jacobi Form. Jacobi form $\varphi(z ; \tau)$ with weight- $k$ and index- $m$ has following transformation formulae [11];

$$
\begin{aligned}
& \varphi\left(\frac{z}{\tau} ;-\frac{1}{\tau}\right)=\tau^{k} \mathrm{e}^{-2 \pi \mathrm{i} m \frac{z^{2}}{\tau}} \varphi(z ; \tau) \\
& \varphi(z ; \tau+1)=\varphi(z+1 ; \tau)=\varphi(z ; \tau) \\
& \varphi(z+\tau ; \tau)=q^{-m} \mathrm{e}^{-4 \pi \mathrm{i} m z} \varphi(z ; \tau)
\end{aligned}
$$

A.3. Theta Functions and Characters. We define the theta function for $a \bmod 2 P$ by (see, e.g., [21] )

$$
\begin{aligned}
\vartheta_{P, a}(z ; \tau) & =\vartheta\left[\begin{array}{c}
\frac{a}{2 P} \\
0
\end{array}\right](2 P z ; 2 P \tau) \\
& =\sum_{n \in \mathbb{Z}} q^{\frac{(2 P n+a)^{2}}{4 P}} \mathrm{e}^{2 \pi \mathrm{i} z(2 P n+a)} .
\end{aligned}
$$

A set of functions $\left\{\vartheta_{P, a}(z ; \tau) \mid a \bmod 2 P\right\}$ spans a $(2 P)$-dimensional space of entire function $f(z)$ such that

$$
\begin{aligned}
& f(z+1)=f(z), \\
& f(z+\tau)=\mathrm{e}^{-4 \pi \mathrm{i} P z-2 \pi \mathrm{i} P \tau} f(z) .
\end{aligned}
$$

The $S$-transformation formula is

$$
\vartheta_{P, a}(z ; \tau)=\sqrt{\frac{\mathrm{i}}{\tau}} \frac{1}{\sqrt{2 P}} \mathrm{e}^{-\pi \mathrm{i} \frac{2 P}{\tau} z^{2}} \sum_{b=0}^{2 P-1} \mathrm{e}^{\frac{a b}{P} \pi \mathrm{i}} \vartheta_{P, b}\left(\frac{z}{\tau} ;-\frac{1}{\tau}\right) .
$$

With a help of this identity, we have

$$
\left(\vartheta_{P, a}-\vartheta_{P,-a}\right)(z ; \tau)=\mathrm{i} \sqrt{\frac{\mathrm{i}}{\tau}} \mathrm{e}^{-2 \pi \mathrm{i} \frac{P}{\tau} z^{2}} \sum_{b=1}^{P-1} \mathbf{S}(P)_{a b}\left(\vartheta_{P, b}-\vartheta_{P,-b}\right)\left(\frac{z}{\tau} ;-\frac{1}{\tau}\right),
$$

where

$$
\mathbf{S}(P)_{a b}=\sqrt{\frac{2}{P}} \sin \left(\frac{a b}{P} \pi\right)
$$

The character of the level- $k \mathrm{SU}(2)$ affine algebra is given by [15]

$$
\chi_{k, \ell}(z ; \tau)=\frac{\vartheta_{k+2,2 \ell+1}-\vartheta_{k+2,-2 \ell-1}}{\vartheta_{2,1}-\vartheta_{2,-1}}(z ; \tau) .
$$

Note that the Macdonald-Weyl denominator identity proves

$$
\left(\vartheta_{2,1}-\vartheta_{2,-1}\right)(z ; \tau)=-\mathrm{i} \theta_{11}(2 z ; \tau) .
$$

We define the weight-3/2 modular form from the $\mathrm{SU}(2)$ affine character as

$$
\frac{\Psi_{P}^{(a)}(\tau)}{[\eta(\tau)]^{3}}=\chi_{P-2, \frac{a-1}{2}}(0 ; \tau)=\frac{\vartheta_{P, a}-\vartheta_{P,-a}}{\vartheta_{2,1}-\vartheta_{2,-1}}(0 ; \tau)
$$


where $a \in \mathbb{Z}$ satisfying $0<a<P$. We see that the modular form has a Fourier transformation as

$$
\Psi_{P}^{(a)}(\tau)=\frac{1}{2} \sum_{n \in \mathbb{Z}} n \psi_{2 P}^{(a)}(n) q^{\frac{n^{2}}{4 P}}
$$

where we have used an odd periodic function

$$
\psi_{2 P}^{(a)}(n)= \begin{cases} \pm 1, & n= \pm a \bmod 2 P \\ 0, & \text { otherwise }\end{cases}
$$

See that, in case $P=2$, we have

$$
\Psi_{2}^{(1)}(\tau)=[\eta(\tau)]^{3}
$$

The modular transformation properties are summarized as

$$
\begin{gathered}
\Psi_{P}^{(a)}(\tau)=\left(\frac{\mathrm{i}}{\tau}\right)^{3 / 2} \sum_{b=1}^{P-1} \mathbf{S}(P)_{a b} \Psi_{P}^{(b)}\left(-\frac{1}{\tau}\right) \\
\Psi_{P}^{(a)}(\tau+1)=\mathrm{e}^{\frac{a^{2}}{2 P} \pi \mathrm{i}} \Psi_{P}^{(a)}(\tau) .
\end{gathered}
$$

\section{Appendix B. Fourier Expansions of the Harmonic MaAss Forms}

We give the Fourier expansion of the harmonic Maass forms $M_{P}^{(a)}(z ; \tau)$ (4.28) at $z \in$ $\left\{\frac{1}{2}, \frac{1+\tau}{2}, \frac{\tau}{2}\right\}$, which are useful in the character decomposition of the elliptic genera in terms of the level $k=P-1$ SCFT. We have directly computed (4.28) using Mathematica and Maple.

- $P=2$

$$
\begin{aligned}
\mu\left(\frac{1}{2} ; \tau\right) & =h_{2}(\tau) \cdot \eta(\tau)=\frac{q^{-\frac{1}{8}}}{4}\left[1+3 q-7 q^{2}+14 q^{3}-21 q^{4}+\cdots\right] \\
\mu\left(\frac{1+\tau}{2} ; \tau\right) & =h_{3}(\tau) \cdot \eta(\tau)=q^{\frac{3}{8}}\left[2-6 q^{\frac{1}{2}}+14 q-28 q^{\frac{3}{2}}+54 q^{2}-98 q^{\frac{5}{2}}+168 q^{3}-\cdots\right] \\
\mu\left(\frac{\tau}{2} ; \tau\right) & =h_{4}(\tau) \cdot \eta(\tau)=q^{\frac{3}{8}}\left[-2-6 q^{\frac{1}{2}}-14 q-28 q^{\frac{3}{2}}-54 q^{2}-98 q^{\frac{5}{2}}-168 q^{3}-\cdots\right]
\end{aligned}
$$


- $P=3$

$$
\begin{aligned}
H_{3}^{(1)}\left(\frac{1}{2} ; \tau\right) & =\frac{q^{-\frac{1}{12}}}{8}\left[3+8 q-25 q^{2}+72 q^{3}-\cdots\right] \\
H_{3}^{(1)}\left(\frac{1+\tau}{2} ; \tau\right) & =q^{\frac{5}{12}}\left[4-20 q^{\frac{1}{2}}+80 q-270 q^{\frac{3}{2}}+812 q^{2}-2228 q^{\frac{5}{2}}+5680 q^{3}-13650 q^{\frac{7}{2}}+\cdots\right] \\
H_{3}^{(1)}\left(\frac{\tau}{2} ; \tau\right) & =q^{\frac{5}{12}}\left[-4-20 q^{\frac{1}{2}}-80 q-270 q^{\frac{3}{2}}-812 q^{2}-2228 q^{\frac{5}{2}}-5680 q^{3}-13650 q^{\frac{7}{2}}-\cdots\right] \\
H_{3}^{(2)}\left(\frac{1}{2} ; \tau\right) & =\frac{q^{-\frac{1}{3}}}{16}\left[1+10 q-20 q^{2}+40 q^{3}+\cdots\right] \\
H_{3}^{(2)}\left(\frac{1+\tau}{2} ; \tau\right) & =q^{\frac{2}{3}}\left[5-32 q^{\frac{1}{2}}+134 q-448 q^{\frac{3}{2}}+1315 q^{2}-3520 q^{\frac{5}{2}}+8764 q^{3}-20608 q^{\frac{7}{2}}+\cdots\right] \\
H_{3}^{(2)}\left(\frac{\tau}{2} ; \tau\right) & =q^{\frac{2}{3}}\left[5+32 q^{\frac{1}{2}}+134 q+448 q^{\frac{3}{2}}+1315 q^{2}+3520 q^{\frac{5}{2}}+8764 q^{3}+20608 q^{\frac{7}{2}}+\cdots\right]
\end{aligned}
$$

- $P=4$

$$
\begin{aligned}
& H_{4}^{(1)}\left(\frac{1}{2} ; \tau\right)=\frac{q^{-\frac{1}{16}}}{64}\left[29+70 q-258 q^{2}+911 q^{3}+\cdots\right] \\
& H_{4}^{(1)}\left(\frac{1+\tau}{2} ; \tau\right)=q^{\frac{7}{16}}\left[6-42 q^{\frac{1}{2}}+238 q-1134 q^{\frac{3}{2}}+4718 q^{2}+59754 q^{\frac{5}{2}}-188480 q^{3}+\cdots\right] \\
& H_{4}^{(1)}\left(\frac{\tau}{2} ; \tau\right)=q^{\frac{7}{16}}\left[-6-42 q^{\frac{1}{2}}-238 q-1134 q^{\frac{3}{2}}-4718 q^{2}-59754 q^{\frac{5}{2}}-188480 q^{3}-\cdots\right] \\
& H_{4}^{(2)}\left(\frac{1}{2} ; \tau\right)=\frac{q^{-\frac{1}{4}}}{8}\left[1+8 q-21 q^{2}+56 q^{3}+\cdots\right] \\
& H_{4}^{(2)}\left(\frac{1+\tau}{2} ; \tau\right)=q^{\frac{3}{4}}\left[14-128 q^{\frac{1}{2}}+762 q-3584 q^{\frac{3}{2}}+14434 q^{2}+51840 q^{\frac{5}{2}}-170212 q^{3}+\cdots\right] \\
& H_{4}^{(2)}\left(\frac{\tau}{2} ; \tau\right)=q^{\frac{3}{4}}\left[14+128 q^{\frac{1}{2}}+762 q+3584 q^{\frac{3}{2}}+14434 q^{2}+51840 q^{\frac{5}{2}}+170212 q^{3}+\cdots\right] \\
& H_{4}^{(3)}\left(\frac{1}{2} ; \tau\right)=\frac{q^{-\frac{9}{16}}}{64}\left[1+21 q-7 q^{2}-77 q^{3}+\cdots\right] \\
& H_{4}^{(3)}\left(\frac{1+\tau}{2} ; \tau\right)=q^{\frac{15}{16}}\left[14-140 q^{\frac{1}{2}}+852 q-3990 q^{\frac{3}{2}}+15836 q^{2}+55890 q^{\frac{5}{2}}-180298 q^{3}+\cdots\right] \\
& H_{4}^{(3)}\left(\frac{\tau}{2} ; \tau\right)=q^{\frac{15}{16}}\left[-14-140 q^{\frac{1}{2}}-852 q-3990 q^{\frac{3}{2}}-15836 q^{2}-55890 q^{\frac{5}{2}}-180298 q^{3}-\cdots\right]
\end{aligned}
$$


- $P=5$

$$
\begin{aligned}
H_{5}^{(1)}\left(\frac{1}{2} ; \tau\right) & =\frac{q^{-\frac{1}{20}}}{128}\left[65+144 q-591 q^{2}+\cdots\right] \\
H_{5}^{(1)}\left(\frac{1+\tau}{2} ; \tau\right) & =q^{\frac{9}{20}}\left[8-72 q^{\frac{1}{2}}+528 q-3252 q^{\frac{3}{2}}+16560 q^{2}-71268 q^{\frac{5}{2}}+\cdots\right] \\
H_{5}^{(1)}\left(\frac{\tau}{2} ; \tau\right) & =q^{\frac{9}{20}}\left[-8-72 q^{\frac{1}{2}}-528 q-3252 q^{\frac{3}{2}}-16560 q^{2}-71268 q^{\frac{5}{2}}-\cdots\right] \\
H_{5}^{(2)}\left(\frac{1}{2} ; \tau\right) & =\frac{q^{-\frac{1}{5}}}{256}\left[46+315 q-980 q^{2}+\cdots\right] \\
H_{5}^{(2)}\left(\frac{1+\tau}{2} ; \tau\right) & =q^{\frac{4}{5}}\left[27-320 q^{\frac{1}{2}}+2468 q-14976 q^{\frac{3}{2}}+77022 q^{2}-349248 q^{\frac{5}{2}}+\cdots\right] \\
H_{5}^{(2)}\left(\frac{\tau}{2} ; \tau\right) & =q^{\frac{4}{5}}\left[27+320 q^{\frac{1}{2}}+2468 q+14976 q^{\frac{3}{2}}+77022 q^{2}+349248 q^{\frac{5}{2}}+\cdots\right] \\
H_{5}^{(3)}\left(\frac{1}{2} ; \tau\right) & =\frac{q^{-\frac{9}{20}}}{128}\left[5+80 q-90 q^{2}-\cdots\right] \\
H_{5}^{(4)}\left(\frac{\tau}{2} ; \tau\right) & =q^{\frac{6}{5}}\left[42+576 q^{\frac{1}{2}}+4614 q+27776 q^{\frac{3}{2}}+138567 q^{2}+597032 q^{\frac{5}{2}}+\cdots\right] \\
H_{5}^{(4)}\left(\frac{1}{2} ; \tau\right) & =\frac{q^{-\frac{4}{5}}}{256}\left[1+36 q+96 q^{2}+\cdots\right] \\
H_{5}^{(3)}\left(\frac{1+\tau}{2} ; \tau\right) & =q^{\frac{21}{20}}\left[48-630 q^{\frac{1}{2}}+4984 q-30072 q^{\frac{3}{2}}+151776 q^{2}-669194 q^{\frac{5}{2}}+\cdots\right] \\
H_{5}^{(3)}\left(\frac{\tau}{2} ; \tau\right) & =q^{\frac{21}{20}}\left[-48-630 q^{\frac{1}{2}}-4984 q-30072 q^{\frac{3}{2}}-151776 q^{2}-669194 q^{\frac{5}{2}}-\cdots\right]
\end{aligned}
$$

\section{REFERENCES}

[1] G. E. Andrews, Mock theta functions, in L. Ehrenpreis and R. C. Gunning, eds., Theta Functions - Bowdoin 1987, vol. 49 (part 2) of Proc. Symp. Pure Math., pp. 283-298, Amer. Math. Soc., Providence, 1989.

[2] K. Bringmann and J. Lovejoy, Dyson's rank, overpartitions, and weak Maass forms, Int. Math. Res. Not. 2007, rnm063 (2007), 34 pages.

[3] K. Bringmann and K. Ono, The $f(q)$ mock theta function conjecture and partition ranks, Invent. Math. 165, 243-266 (2006).

[4] F. J. Dyson, Some guesses in the theory of partitions, Eureka (Cambridge) 8, 10-15 (1944). 
[5] T. Eguchi, H. Ooguri, A. Taormina, and S.-K. Yang, Superconformal algebras and string compactification on manifolds with $S U(n)$ holonomy, Nucl. Phys. B 315, 193221 (1989).

[6] T. Eguchi, Y. Sugawara, and A. Taormina, Liouville field, modular forms and elliptic genera, JHEP 2007, 119 (2007), 21 pages.

[7] —, Modular forms and elliptic genera for ALE spaces, arXiv:0803.0377 (2008).

[8] T. Eguchi and A. Taormina, Unitary representations of the $N=4$ superconformal algebra, Phys. Lett. B 196, 75-81 (1986).

[9] - Character formulas for the $N=4$ superconformal algebra, Phys. Lett. B 200, 315-322 (1988).

[10] - On the unitary representations of $N=2$ and $N=4$ superconformal algebras, Phys. Lett. B 210, 125-132 (1988).

[11] M. Eichler and D. Zagier, The Theory of Jacobi Forms, vol. 55 of Progress in Mathematics, Birkhäuser, Boston, 1985.

[12] K. Hikami, Quantum invariant for torus link and modular forms, Commun. Math. Phys. 246, 403-426 (2004).

[13] - Mock (false) theta functions as quantum invariants, Regular \& Chaotic Dyn. 10, 509-530 (2005).

[14] - On the quantum invariant for the spherical Seifert manifold, Commun. Math. Phys. 268, 285-319 (2006).

[15] V. G. Kac, Infinite Dimensional Lie Algebras, Cambridge Univ. Press, Cambridge, 1990, 3rd ed.

[16] T. Kawai, Y. Yamada, and S.-K. Yang, Elliptic genera and $N=2$ superconformal field theory, Nucl. Phys. B 414, 191-212 (1994).

[17] K. Miki, The representation theory of the SO(3) invariant superconformal algebra, Int. J. Mod. Phys. A 5, 1293-1318 (1990).

[18] L. J. Mordell, The definite integral $\int_{-\infty}^{\infty} \frac{e^{a x^{2}+b x}}{e^{c x}+d} d x$ and the analytic theory of numbers, Acta Math. 61, 323-360 (1933).

[19] M. Nieper-Wißkirchen, Chern Numbers and Rozansky-Witten Invariants of Compact Hyper-Kähler Manifolds, World Scientific, 2003.

[20] A. M. Semikhatov, I. Y. Tipunin, and A. Taormina, Higher-level Appell functions, modular transformations, and characters, Commun. Math. Phys. 255, 469-512 (2005).

[21] G. Shimura, On modular forms of half integral weight, Ann. Math. 97, 440-481 (1973).

$[22]$ N. J. A. Sloane, On-line encyclopedia of integer sequences, http://www.research.att.com/ njas/sequences/index.html.

[23] A. Taormina, Liouville theory and elliptic genera, arXiv:0808.2376 (2008).

[24] G. N. Watson, The final problem: an account of the mock theta functions, J. London Math. Soc. 11, 55-80 (1936).

[25] E. Witten, Elliptic genera and quantum field theory, Commun. Math. Phys. 109, 525536 (1987). 
[26] D. Zagier, Ramanujan's mock theta functions and their applications [d'après Zwegers and Bringmann-Ono], Séminaire Bourbaki 986 (2006-2007).

[27] S. P. Zwegers, Mock Theta Functions, Ph.D. thesis, Universiteit Utrecht (2002).

Yukawa Institute for Theoretical Physics, Kyoto University, Kyoto 606-8502, Japan

E-mail address: eguchi@yukawa.kyoto-u.ac.jp

Department of Mathematics, Naruto University of Education, Tokushima 772-8502, JAPAN.

E-mail address: hikami@naruto-u.ac.jp 